Supplementary Information

\title{
Optical Anisotropy and Phase Transitions in Lead Halide Perovskites
}

\author{
Feifan Wang, Lucas Huber, Sebastian F. Maehrlein, X.-Y. Zhu* \\ Department of Chemistry, Columbia University, New York, NY 10027, USA \\ *Correspondence and requests for materials should be addressed to X.-Y.Z. (email: xyzhu@columbia.edu)
}

\section{METHODS}

Sample preparation and characterization. The growth of lead bromide perovslite crystals were based on our previous published method. ${ }^{1} \mathrm{CsPbBr}_{3}$ single crystals were synthesized from the precursor solution of $\mathrm{CsBr}$ and $\mathrm{PbBr}_{2}$ with a molar ratio of $1: 1$ in dimethylsulfoxide. The solution was titrated with methanol and further stirred at $50{ }^{\circ} \mathrm{C}$ until a yellow permanent suspension was formed. Methanol was used as the anti-solvent for slow vapor diffusion growth method. The $\mathrm{MAPbBr}_{3}$ single crystals were prepared from the precursor solution of $\mathrm{MABr}$ and $\mathrm{PbBr}_{2}$ with 1:1 molar ratio in N,N-dimethylformamide. A mixture of dichloromethane and nitromethane was used as the anti-solvent for $\mathrm{MAPbBr}_{3}$ crystal growth. For the $\mathrm{MA}_{\mathrm{x}} \mathrm{Cs}_{1-\mathrm{x}} \mathrm{PbBr}_{3}$ crystals, $\mathrm{MABr}$ was added into the precursor solution with molar ratio to $\mathrm{CsBr}$ of 0.10 and 0.20 .

Two-dimensional optical Kerr effect spectroscopy. The fundamental ultrafast pulse (1.55 $\mathrm{eV}, 0.8 \mathrm{~mJ} /$ pulse, pulse duration $\sim 35 \mathrm{fs}$ ) was delivered by a $10 \mathrm{kHz}$ Ti:sapphire regenerative amplifier (Legend, Coherent Inc.). The broadband pump pulse (centered at $\sim 2.25 \mathrm{eV}$ ) was generated from a homebuilt noncolinear optical parametric amplifier (NOPA) pumped by the second harmonic of the fundamental. The probe pulse was from the attenuated fundamental at pulse energy $<200 \mathrm{~nJ}$. The collinear double pulse (35 $\pm 5 \mu \mathrm{J}$ per pair, $\sim 30 \mathrm{fs})$ was created by a pulse shaper (PhaseTech) with active dispersion correction. The pump spot size of $160 \mu \mathrm{m}$ on the sample was set to be much larger than the probe spot $(30 \mu \mathrm{m})$ to ensure homogeneous excitation. The rotating frame method was used to reduce the number of sampling while maintaining the 
frequency resolution of the pump. The phase cycling was also applied to separate the nonlinear 2D signal from other linear or nonlinear signals (e.g. transient absorption, scattering and OKE caused by one of the pump pulses). ${ }^{2}$ A balanced detection scheme was implemented to detect small $\left(\sim 10^{-}\right.$

${ }^{4}$ ) changes in the ellipticity or linear polarization of the probe. Due to the collinear geometry of the pulse shaper, the absorptive signal was automatically obtained by the real part of the Fourier transform of the 2D signal, which contains both the rephasing and non-rephasing signals. ${ }^{3}$ A liquid nitrogen cooled cryostat (SuperTran, Janis) was used in the temperature dependence experiments with temperature fluctuation of $<0.2 \mathrm{~K}$.

Low-frequency Raman and photoluminescence spectroscopy. Both the Raman and PL measurements were carried out on a home-built inverted microscope (Nikon, Elipse TE300) with a $40 \times$ objective and detected by a spectrometer (SPEX 270M) and a liquid- $\mathrm{N}_{2}$ cooled CCD camera (Princeton Instruments). In the Raman experiments, a $632.8 \mathrm{~nm} \mathrm{CW}$ laser was first filtered with a volume Bragg grating (VBG) beam-splitter and then focused onto the sample surface with power density of $3 \mathrm{~mW} \cdot \mu \mathrm{m}^{-2}$. The Raman shifted signal was collected and purified from the Rayleigh scattering by two ultra-narrowband VBG notch filters. For the PL spectra, a $450 \mathrm{~nm}$ CW laser was used to excite the sample at power density of $0.6 \mu \mathrm{W} \mu \mathrm{m}^{-2}$. In all measurements, the sample was in a cryostat (Oxford Instruments Microstat HiRes2) under vacuum of $10^{-6}$ Torr with temperature fluctuation of $<0.1 \mathrm{~K}$.

Single-crystal X-ray diffraction (SCXRD) experiment was performed on the Agilent SuperNova instrument with an MiTeGen Micro-mount and Mo Ka radiation. The sample temperature was controlled by the Oxford-Diffraction Cryojet. The CrysAlis Pro software package was used for data collection, integration, correction and refinement.

Scanning electron microscopy. The SEM images were collected on a Zeiss Sigma VP SEM with imaging resolution of $5 \mathrm{~nm}$. The energy-dispersion X-ray spectroscopy (EDS) results were obtained from a detector (Bruker XFlash) through the Zeiss SEM and analyzed in the Quantax Esprit software.

\section{Supplementary Figures}



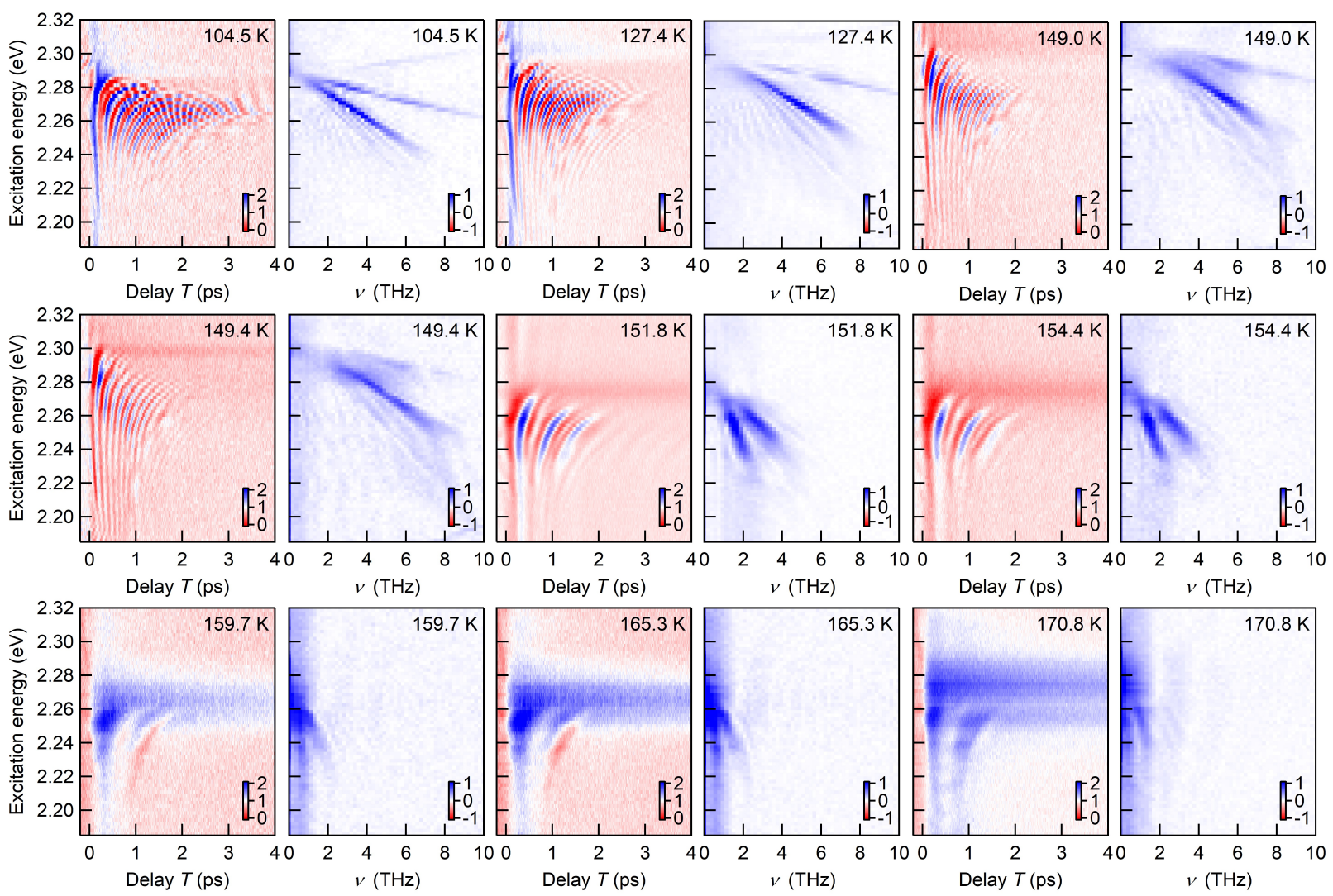

Figure S1. Temperature-dependent time-/frequency-domain 2D-OKE spectra of $\mathrm{MAPbBr}_{3}$ single crystal. Orthorhombic phase: 104.5, 127.4, 149.0, $149.4 \mathrm{~K}$; incommensurate phase: 151.8, 154.4 $\mathrm{K}$; tetragonal phase: $159.7,165.3,170.8 \mathrm{~K}$. The color scale is in arbitrary unit. Within orthorhombic phase, the steepness of the time-domain oscillation discontinuity increases with decreasing the temperature, indicating the sharpening in group index dispersion near band edge at lower temperatures. The birefringence $\Delta n$ is the difference between the refractive index along the slow $\left(n_{\mathrm{s}, \mathrm{pu}}\right)$ and fast $\left(n_{\mathrm{f}, \mathrm{pu}}\right)$ axis, i.e., the $c$ or $a$ crystal axis in our experimental geometry. The fitting is based on the Eq. 1 and 2 in the main text and the Supplementary Note 1. 


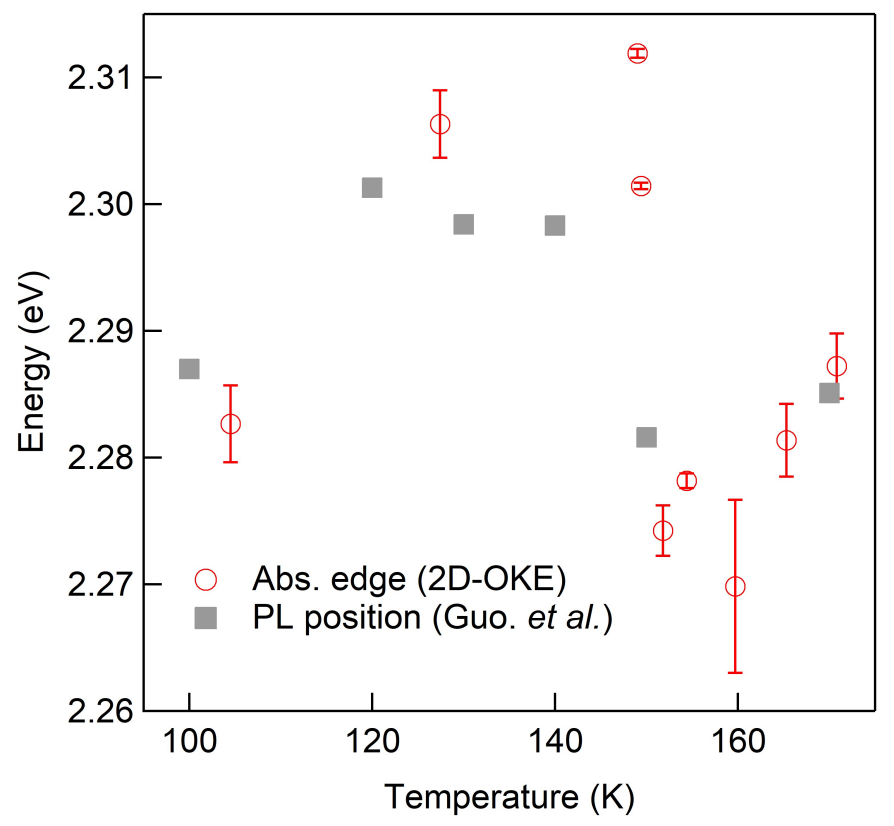

Figure S2. Absorption edge extracted from 2D-OKE and PL peak position from previous work ${ }^{4}$ as a function of temperature for $\mathrm{MAPbBr}_{3}$. The error bars show the standard deviation of the absorption edge identification. Both data exhibit energy reduction near the orthorhombic-toincommensurate phase transition. 

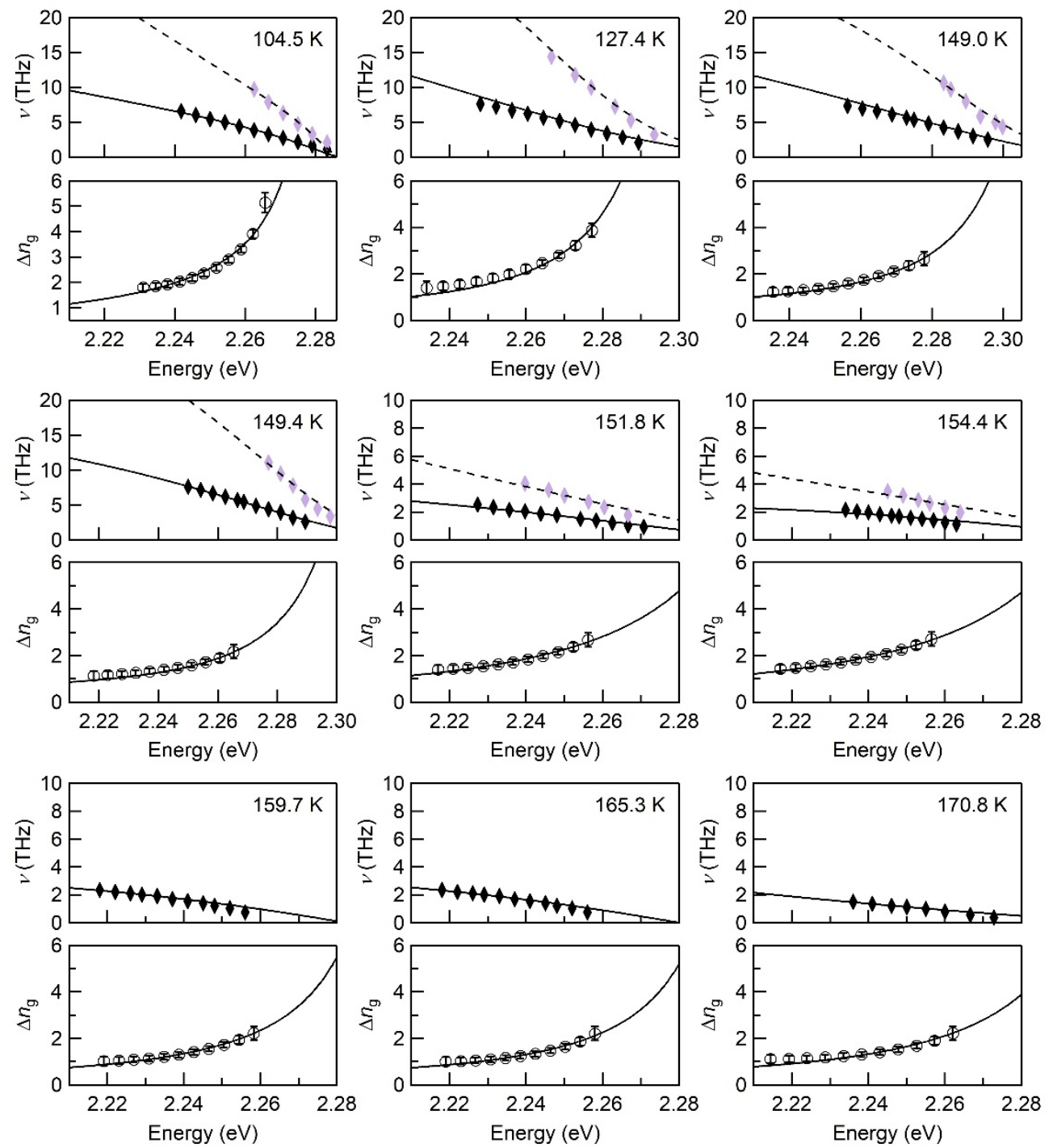

Figure S3. Global fitting results of the observed oscillation frequency and group index mismatch of $\mathrm{MAPbBr}_{3}$ at different temperatures. The diamond markers in upper panels are extracted from the frequency-domain 2D-OKE spectra and the circles in lower panels are from the discontinuity in the time-domain spectra. The fitting output parameters are used to generate the refractive indices and the birefringences of $\mathrm{MAPbBr}_{3}$ at different temperatures. 

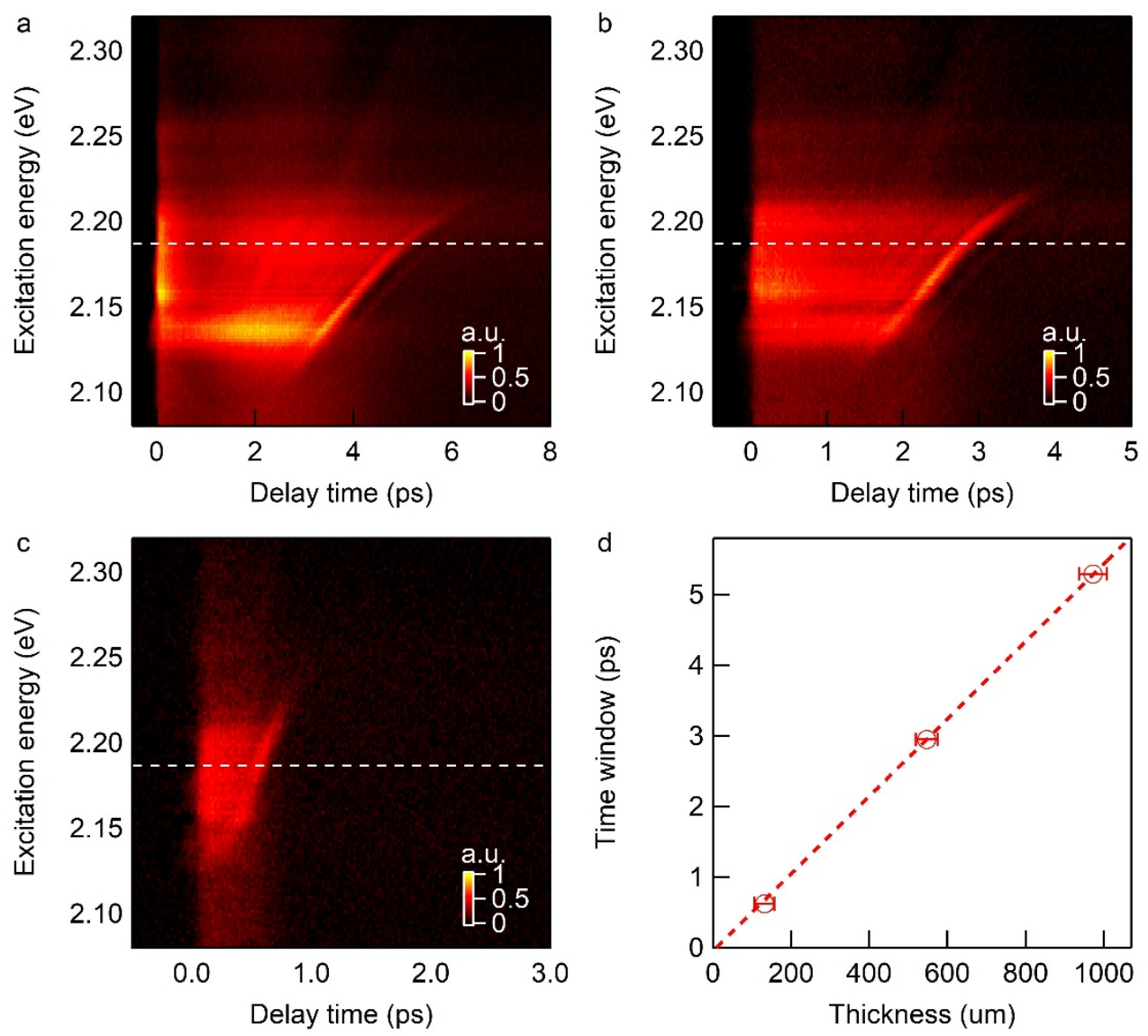

Figure S4. Thickness-dependent 2D-OKE results on $\mathrm{MAPbBr}_{3}$ at 293.0 K. Time-domain 2D-OKE spectra of $\mathrm{MAPbBr}_{3}$ single crystals with thickness of $972 \pm 35 \mu \mathrm{m}$ (a), $547 \pm 28 \mu \mathrm{m}$ (b) and $132 \pm$ $25 \mu \mathrm{m}$ (c). Isotropic signals without oscillation are shown for the cubic phase due to the absence of birefringence. The thickness is measured using a microscope with a calibration ruler. The dashed line represents the excitation energy cut at $2.187 \mathrm{eV}$. (d) Time window of the dispersive signal at $2.187 \mathrm{eV}$ as a function of the sample thickness. Fitting results: $y=a+b^{*} x ; a=-0.04843 \pm 0$. $03862, \mathrm{~b}=0.00548 \pm 0.00007, R^{2}=0.99953$. The linear relationship consolidates the propagation nature of the $3^{\text {rd }}$ order polarization in $2 \mathrm{D}-\mathrm{OKE}$ results. 


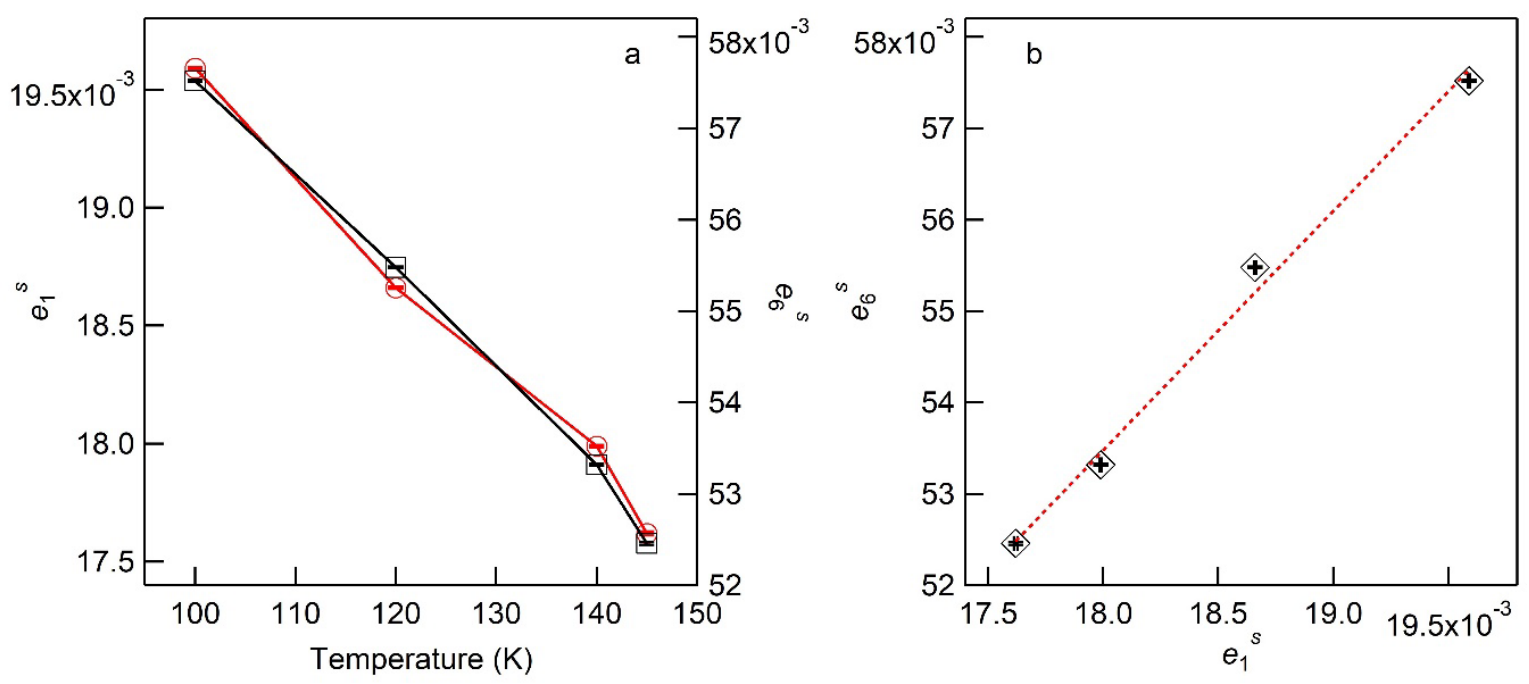

Figure S5. (a) Spontaneous strain tensor elements as a function of temperature of $\mathrm{MAPbBr}_{3}$. (b) Relationship between $e_{1}^{\mathrm{s}}$ and $e_{6} \mathrm{~s}$. Fitting results: $\mathrm{y}=\mathrm{a}+\mathrm{b}^{*} \mathrm{x} ; \mathrm{a}=0.00635 \pm 0.00286, \mathrm{~b}=2.618 \pm$ $0.155, R^{2}=0.993$. It shows a near proportionality between $e_{1}{ }^{\mathrm{s}}$ and $e_{6} \mathrm{~s}$. 

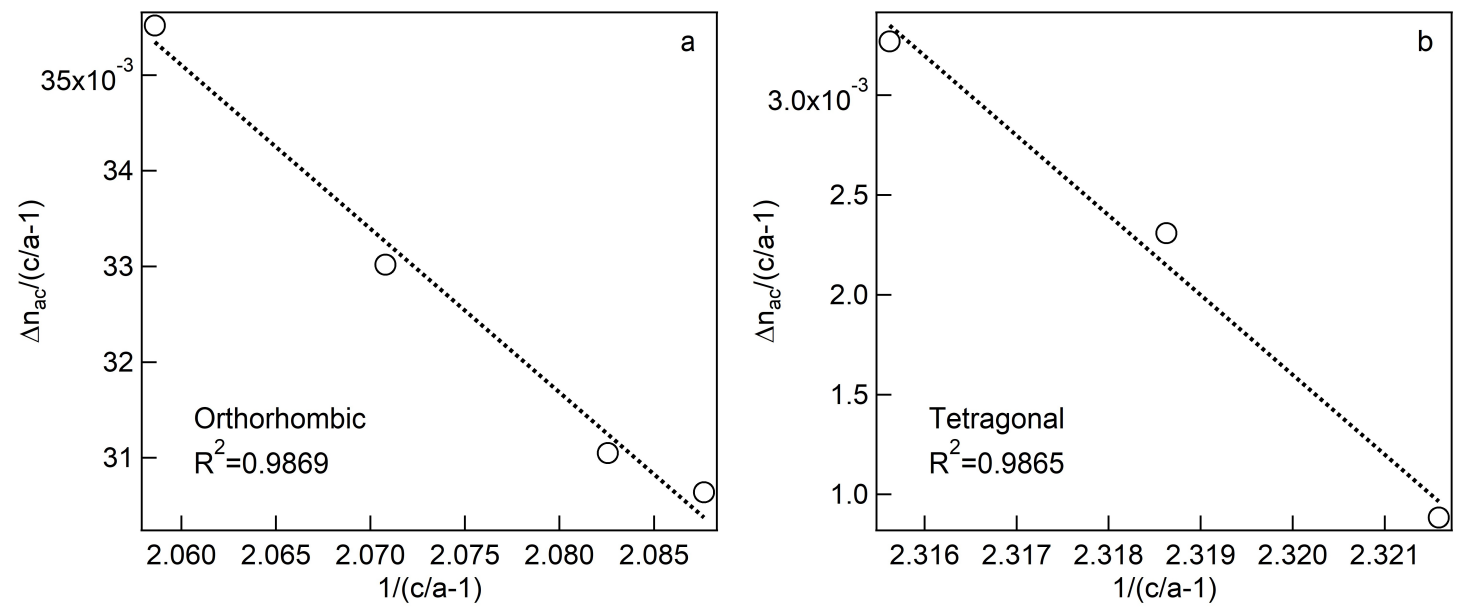

Figure S6. Birefringence extracted from 2D-OKE as a function of lattice parameter contrast c/a-1 in orthorhombic (a) and tetragonal (b) phases. The $\Delta n_{\mathrm{ac}}(2.215 \mathrm{eV})$ was linearly interpolated to the temperatures of the SC-XRD measurements based on Fig. 2d. The linear fitting functions of

$$
\begin{aligned}
& \mathrm{y}=(0.387 \pm 0.029)+(-0.171 \pm 0.014) x \\
& \mathrm{y}=(0.929 \pm 0.109)+(-0.400 \pm 0.047) x
\end{aligned}
$$

build up the correlation between birefringence and lattice structure of $\mathrm{MAPbBr}_{3}$ in the orthorhombic and tetragonal phase, respectively. 


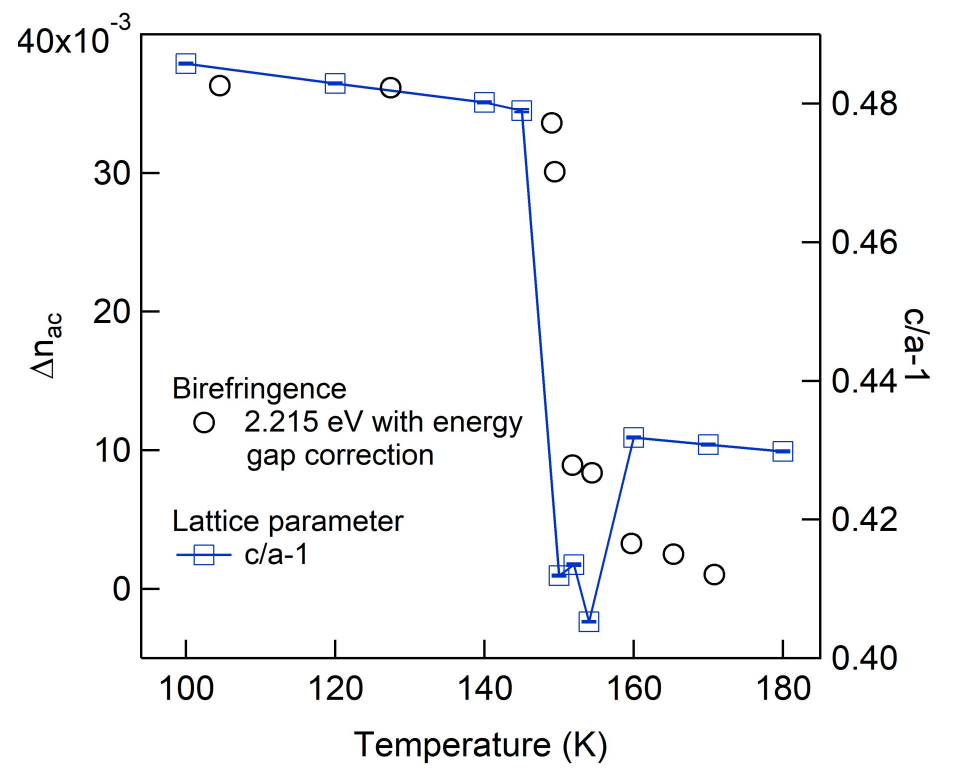

Figure S7. Energy-gap corrected birefringence and lattice parameter contrast as a function of temperature of $\mathrm{MAPbBr}_{3}$. The energy correction is calculated from the difference between the absorption edge of $159.7 \mathrm{~K}$ and that of other temperatures in Fig. S3. The birefringence is taken at the energy of $2.215 \mathrm{eV}$ minus the correction value for each temperature. A correlation between the energy-gap corrected birefringence and the lattice parameter contrast is established. 

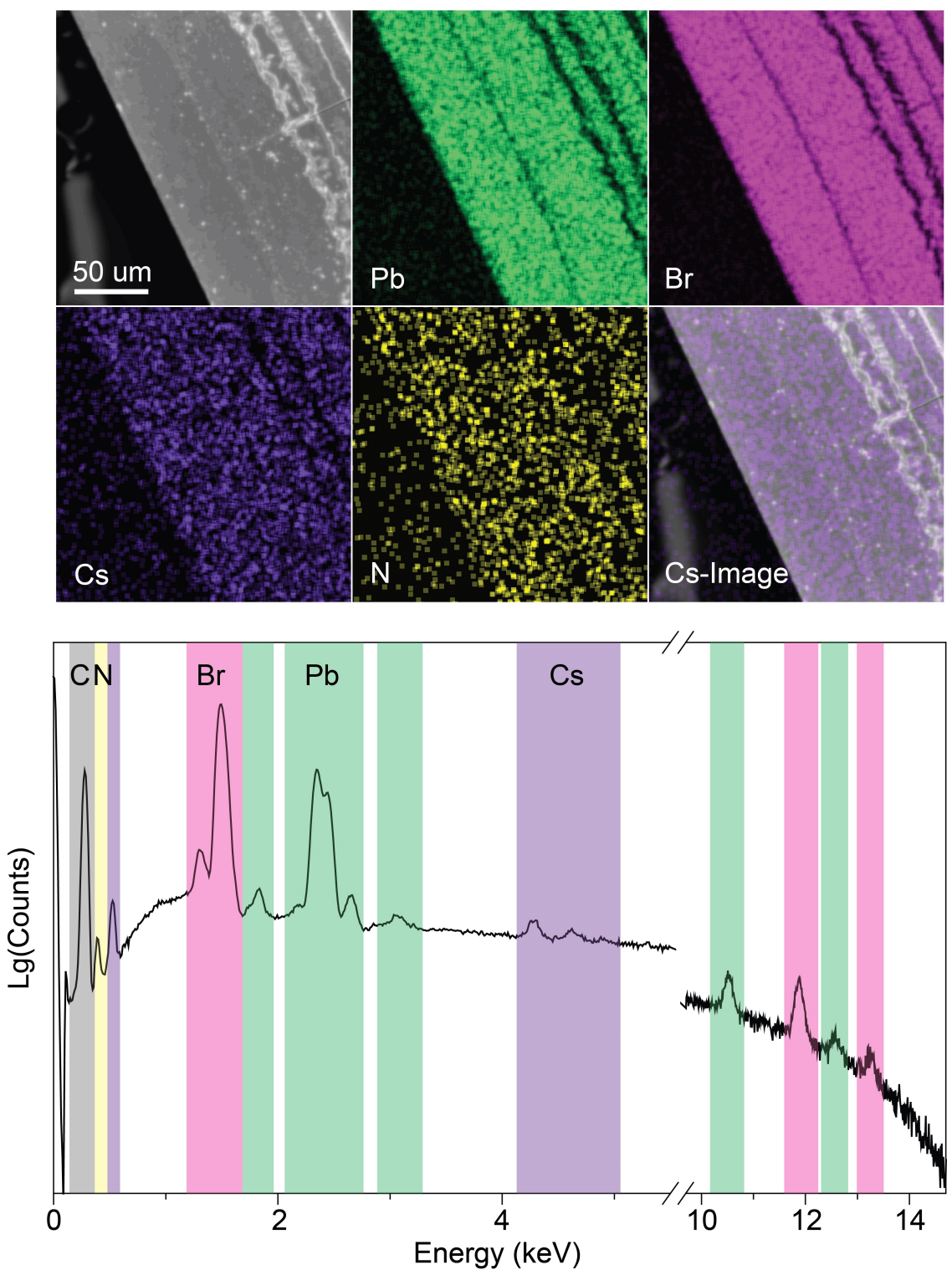

Figure S8. Energy-dispersive X-ray spectroscopy (bottom) and imaging (top) of mixed-cation $\mathrm{Cs}_{\mathrm{x}} \mathrm{MA}_{1-\mathrm{x}} \mathrm{PbBr}_{3}$ single crystal. It is reported that mixing $\mathrm{Cs}^{+}$into $\mathrm{MAPbBr}_{3}$ can induce the phase segregation due to the different crystallization kinetics of the cation species in the solution. ${ }^{1}$ In this work, we select the samples without chemical composition heterogeneity. The EDX imaging shows that the $\mathrm{Cs}$ element is homogeneously distributed in the single crystal. The $\mathrm{Cs} / \mathrm{Pb}$ atomic ratio is determined to be 0.074 from the integrated peak intensity in the EDX spectrum, in agreement with the ratio of 0.07 from the ICP-AES results. 

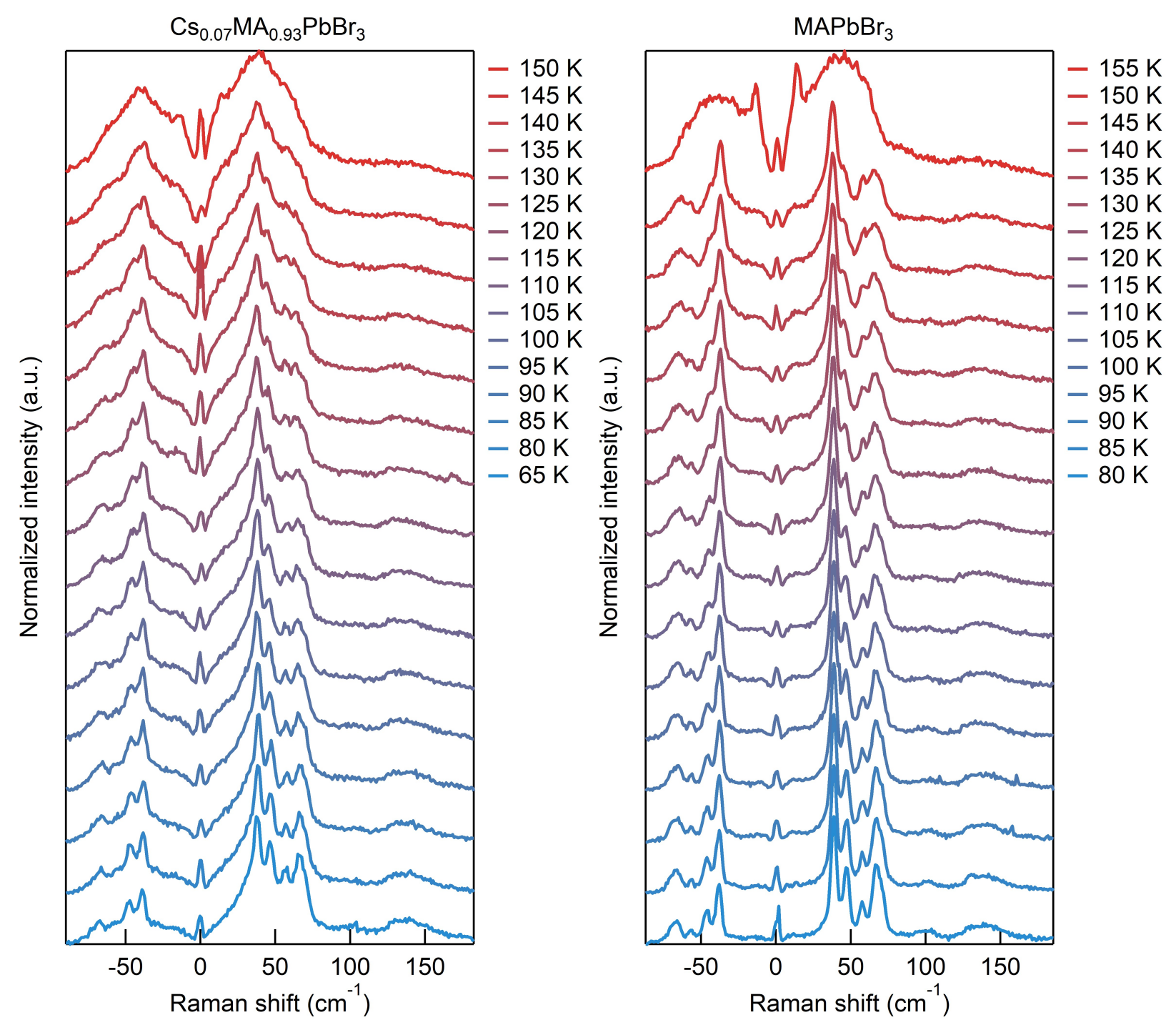

Figure S9. Low-frequency Raman spectra of $\mathrm{Cs}_{0.07} \mathrm{MAA}_{0.93} \mathrm{PbBr}_{3}$ and $\mathrm{MAPbBr}_{3}$ crystals at temperatures moving towards phase transition from the orthorhombic phase. Sharp peaks are shown in both cases at the frozen temperatures, however, the behaviors of the transition are different: in $\mathrm{MAPbBr}_{3}$, the well-defined vibrational peaks suddenly disappear and a broad band takes over at $155 \mathrm{~K}$; while in $\mathrm{Cs}_{0.07} \mathrm{MA}_{0.93} \mathrm{PbBr}_{3}$, the peaks gradually merge from one phase to another. The intensity of the strongest peak at $\sim 39 \mathrm{~cm}^{-1}$ is used as an order parameter to signify the phase transitions. 

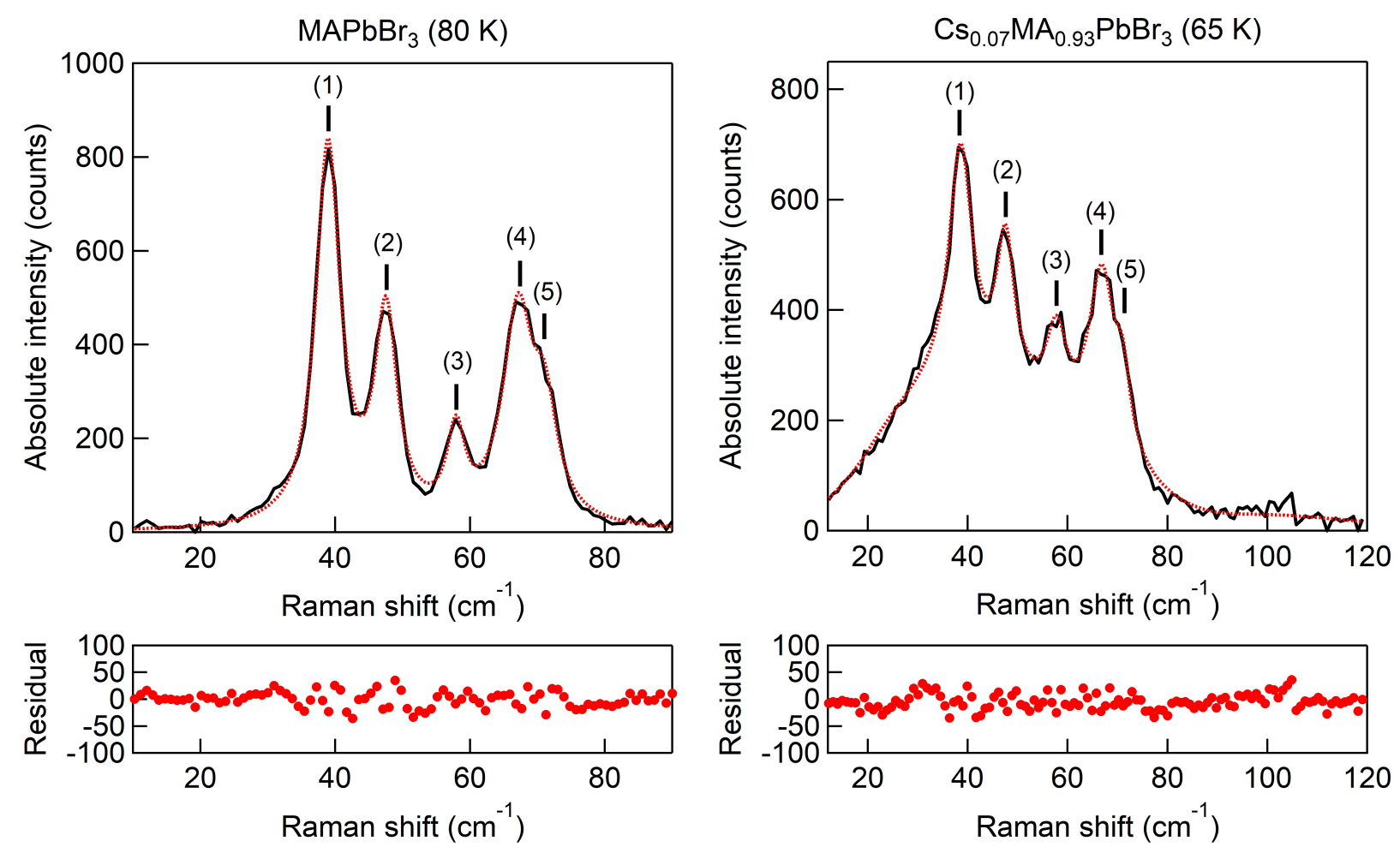

Figure S10. Fitting of the low-frequency Raman spectra of $\mathrm{MAPbBr}_{3}$ and $\mathrm{Cs}_{0.07} \mathrm{MA}_{0.93} \mathrm{PbBr}_{3}$ single crystals. A model containing five damped Lorentz oscillators and a broad relaxation background is used. The bottom panels show the regular residual from the fitting. The types of Raman modes are labeled as (1) octahedra twist mode, (2) octahedra twist mode, (3) octahedra distortion mode, (4) nodding donkey mode around the nitrogen atom, (5) lurching mode of MA, respectively. ${ }^{5}$ 


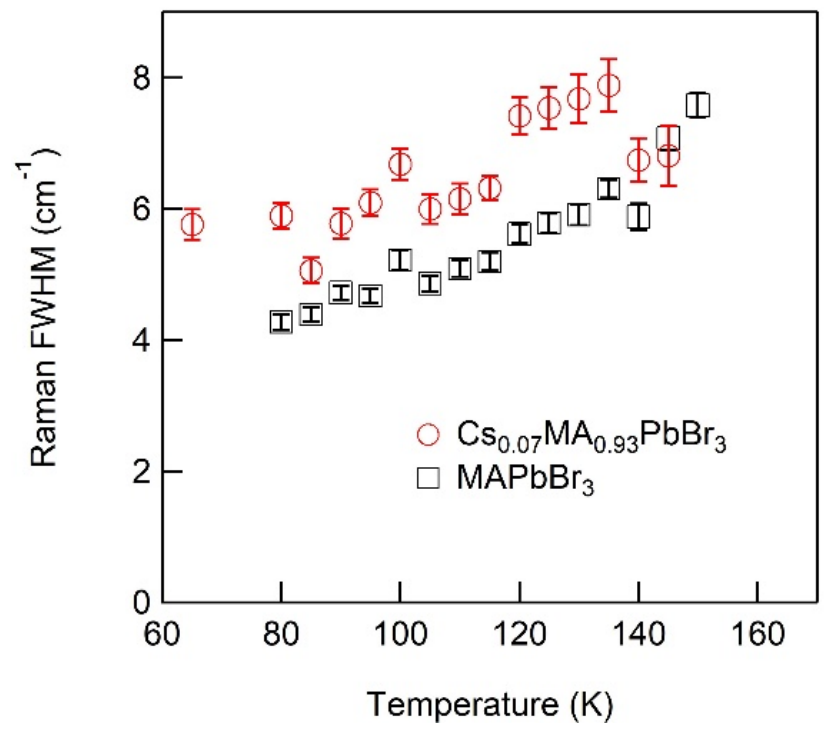

Figure S11. Extracted full width at half maximum of the Raman peak around $39 \mathrm{~cm}^{-1}$ for $\mathrm{Cs}_{0.07} \mathrm{MA}_{0.93} \mathrm{PbBr}_{3}$ and $\mathrm{MAPbBr} 3$ single crystals. 

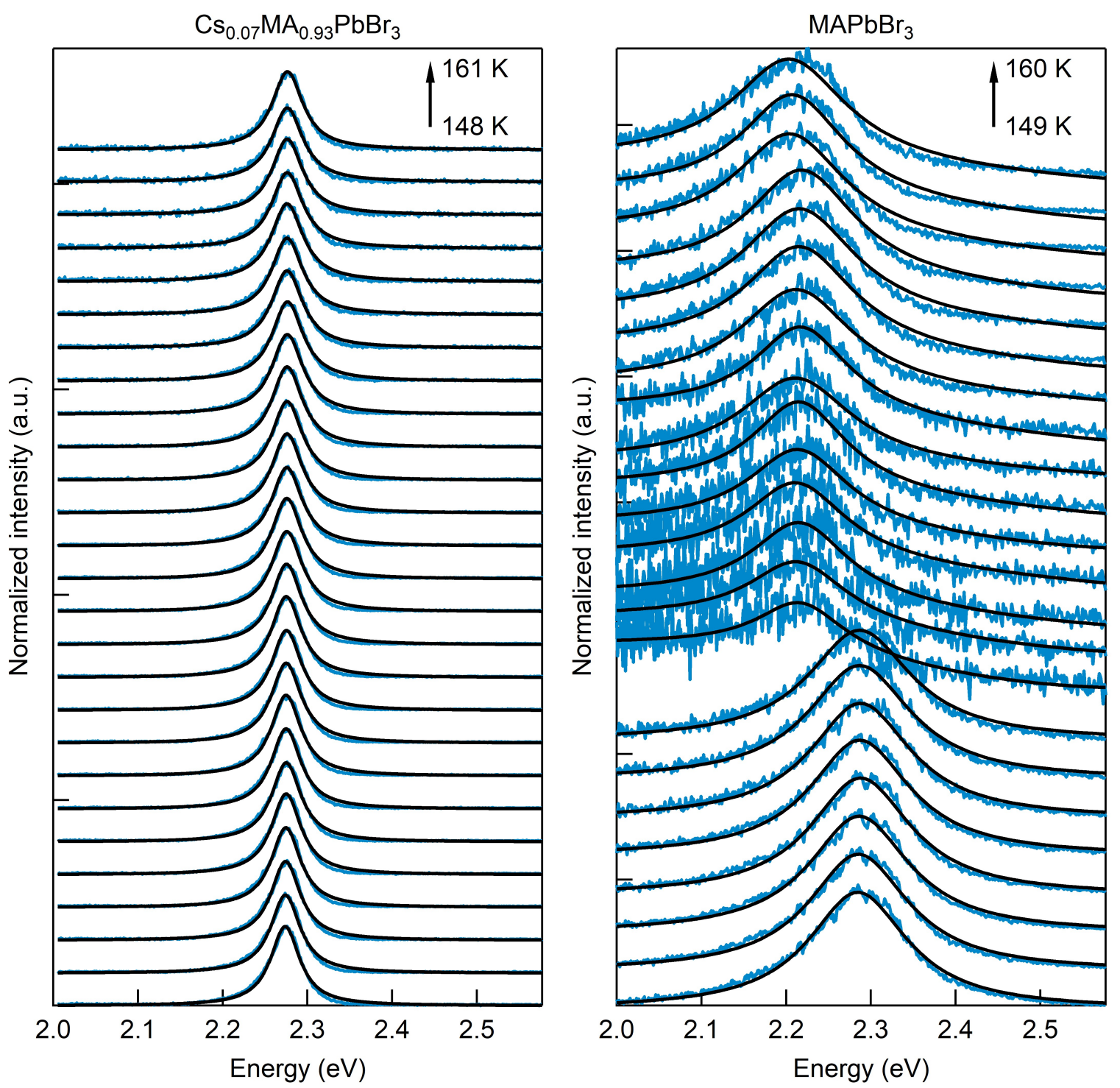

Figure S12. Temperature-dependent photoluminescence spectra of $\mathrm{Cs}_{0.07} \mathrm{MA}_{0.93} \mathrm{PbBr}_{3}$ and $\mathrm{MAPbBr}_{3}$ single crystals. The temperature step is $0.5 \mathrm{~K}$ for both spectra. Black lines show the fitting using a single Lorentz oscillator with background correction. Under the same experimental condition, the photoluminescence efficiency of $\mathrm{Cs}_{0.07} \mathrm{MA}_{0.93} \mathrm{PbBr}_{3}$ is higher than that of $\mathrm{MAPbBr}_{3}$ based on the signal-to-noise ratio. This is consistent with the previous report ${ }^{6}$ that the cationmixing improves the external quantum yield of the light-emitting diode due to the suppression of non-radiative recombination and a longer PL lifetime is observed. 

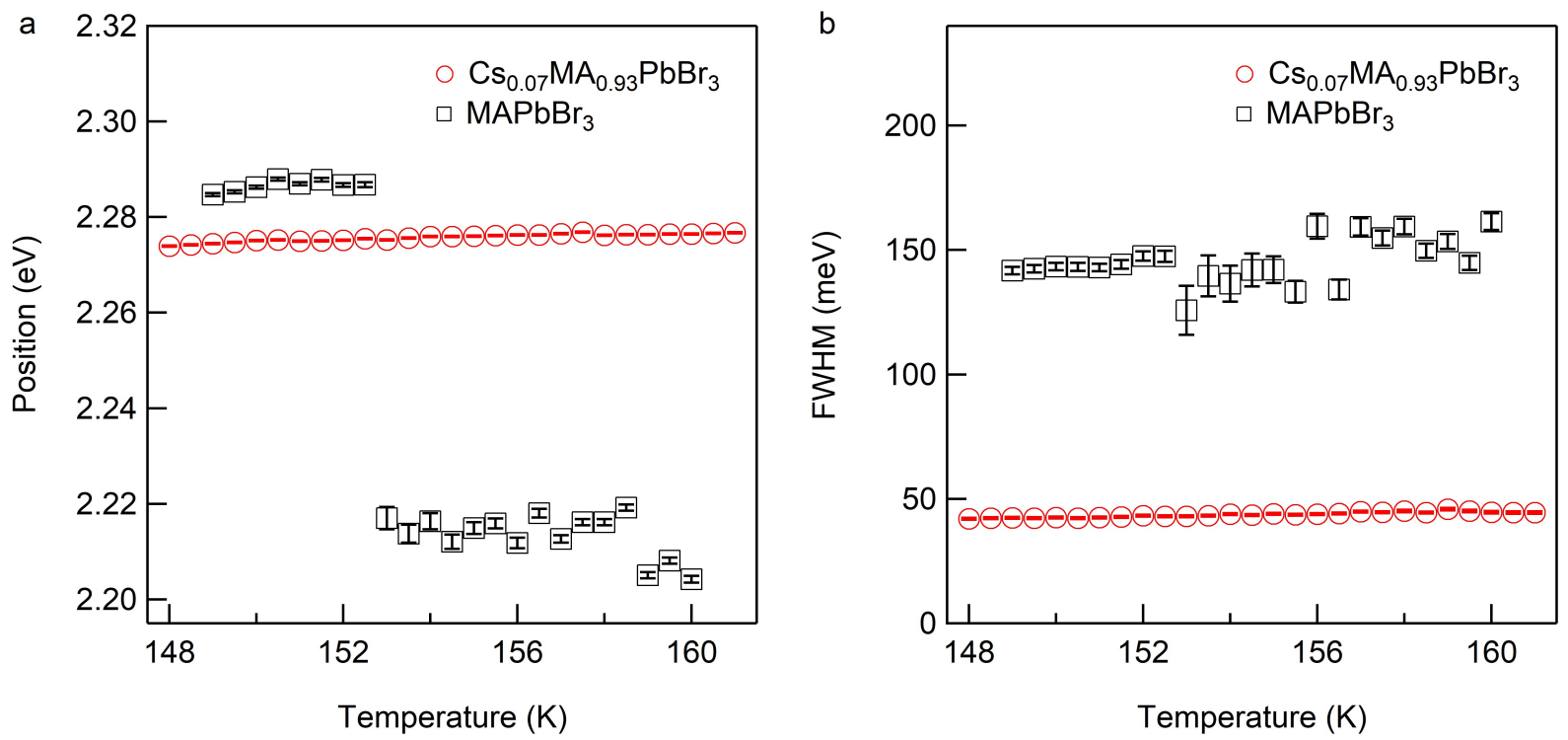

Figure S13. PL peak energy and full-width at half-maximum (FWHM) at different temperatures of $\mathrm{Cs}_{0.07} \mathrm{MA}_{0.93} \mathrm{PbBr}_{3}$ and $\mathrm{MAPbBr}_{3}$ single crystals. The energy gap anomaly is observed at both ends of the incommensurate phase in $\mathrm{MAPbBr}_{3}$, however, it is absent in the mixed-cation sample, indicating different orders of phase transition. The PL peak width of the $\mathrm{Cs}_{0.07} \mathrm{MA}_{0.93} \mathrm{PbBr}_{3}$ is much narrower than that of the $\mathrm{MAPbBr}_{3}$, suggesting the electronic band structure changes in the mixedcation perovskite. 

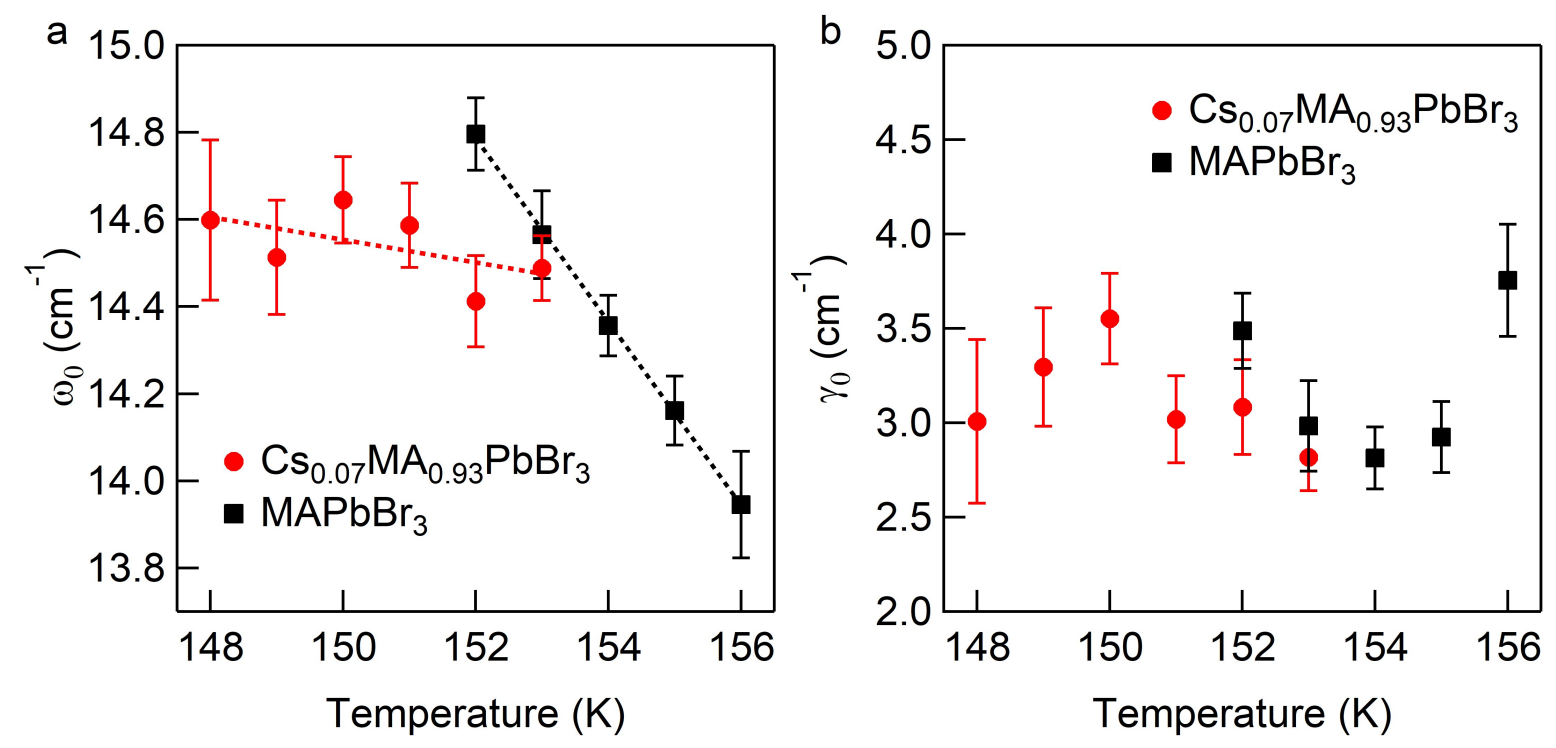

Figure S14. Amplitudon frequency (a) and damping (b) of $\mathrm{Cs}_{0.07} \mathrm{MA}_{0.93} \mathrm{PbBr}_{3}$ and $\mathrm{MAPbBr}_{3}$ single crystals obtained from the low-frequency Raman spectroscopy. The thermal coefficients are extracted by a linear fitting on the amplitudon frequency with functions of $\omega_{0}=(18.468 \pm 2.810)$ $+(-0.026 \pm 0.019) * T$ and $\omega_{0}=(46.771 \pm 0.507)+(-0.210 \pm 0.003) * T$ for $\mathrm{Cs}_{0.07} \mathrm{MA}_{0.93} \mathrm{PbBr}_{3}$ and $\mathrm{MAPbBr}_{3}$, respectively. The amplitudon damping rate of the pure and mixed-cation perovskites is on the same order. 

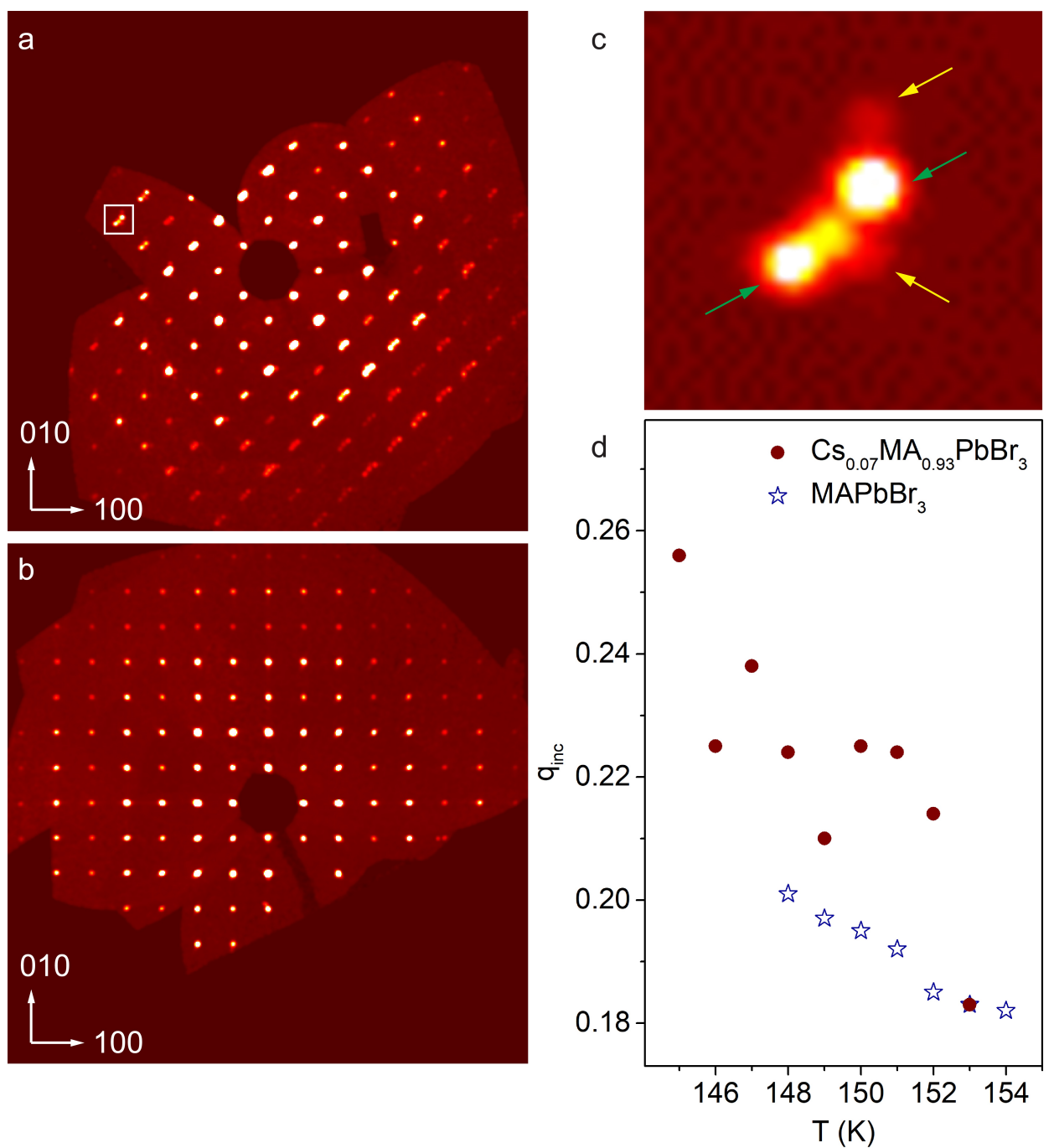

Figure S15. Single-crystal X-ray diffraction patterns of $\mathrm{Cs}_{0.07} \mathrm{MA}_{0.93} \mathrm{PbBr}_{3}$ (a) and $\mathrm{MAPbBr}_{3}$ (b) crystals at 145 K. (c) Magnified reflection pattern of the white box area in (a). The two green arrows assign the reflection from the twin structure and the two yellow arrows represent the satellite reflection due to the incommensurate modulation. (d) Incommensurate $q$-vector of $\mathrm{Cs}_{0.07} \mathrm{MA}_{0.93} \mathrm{PbBr}_{3}$ and $\mathrm{MAPbBr}_{3}$ as a function of temperature. At $145 \mathrm{~K}$, no incommensurate satellite is observed in the diffraction pattern of the pure $\mathrm{MAPbBr}$. 
a

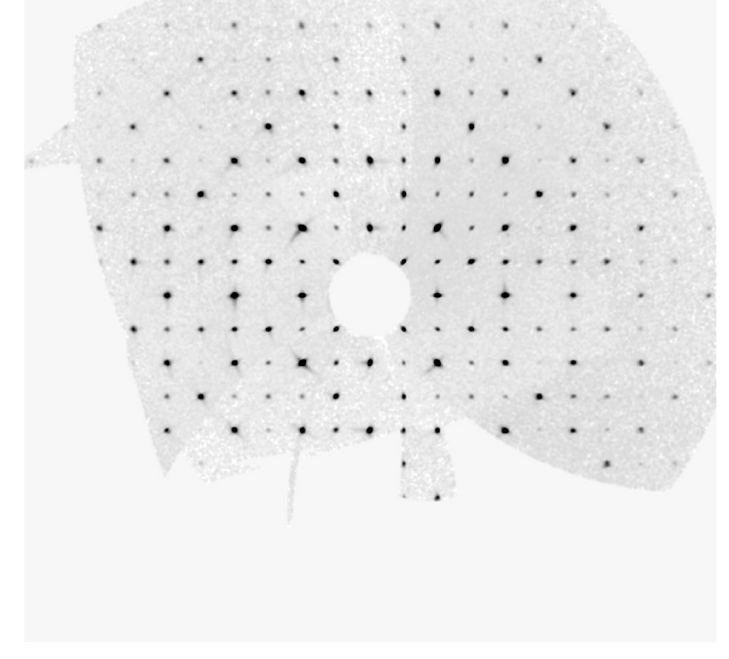

C

Figure S16. Section $(h k 0)$ reflections from single-crystal X-ray diffraction of $\mathrm{CsPbBr}_{3}(\mathrm{a})$, $\mathrm{MA}_{0.10} \mathrm{Cs}_{0.90} \mathrm{PbBr}_{3}$ (b) and $\mathrm{MA}_{0.20} \mathrm{Cs}_{0.80} \mathrm{PbBr}_{3}$ (c) single crystals. No structural heterogeneity is shown in all mixed-cation crystals. (d) Lattice parameters as a function of $\mathrm{MA}^{+}$content. The mixed samples show larger $a$, similar $b$ and smaller $c$ compared with the pure $\mathrm{CsPbBr}_{3}$, indicating a structural transform towards a pseudo-cubic lattice in the mixed samples. Error bars in (d) are based on repeated experiments on different crystals. 

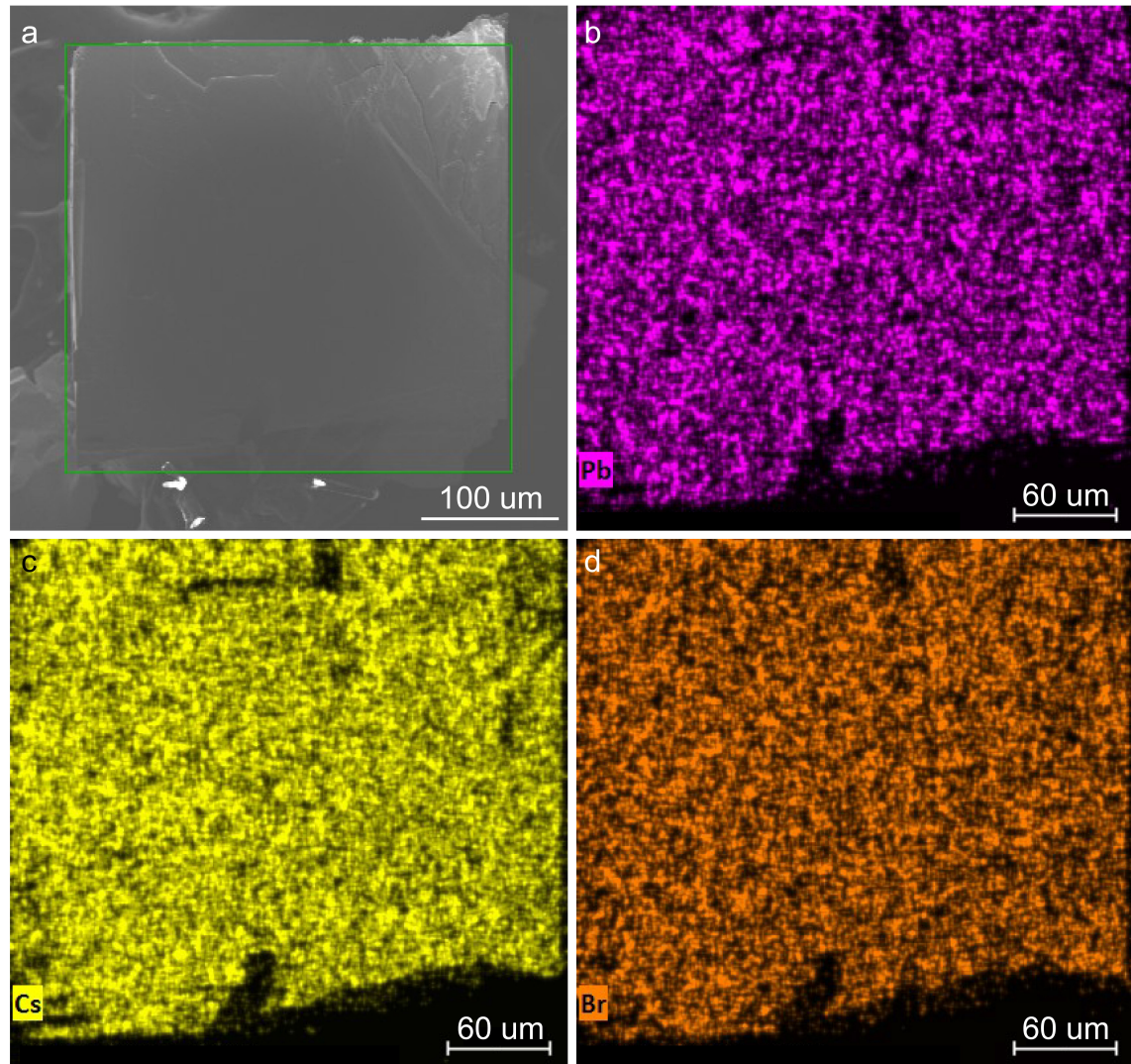

Figure S17. SEM image (a) and element mapping (b-d) of mixed-cation $\mathrm{MA}_{0.16} \mathrm{Cs}_{0.84} \mathrm{PbBr}_{3}$ single crystal. (a) shows the cleaved surface at the position where the 2D-OKE spectra are measured. The green square labels the selected area for the EDS. It indicates the homogeneous distribution of the alloyed cations. The $\mathrm{Cs} / \mathrm{Pb}$ atomic ratio from the integrated peak intensity in the EDX spectrum is used to calculate the $\mathrm{MA}^{+}$cation contents of $16.4 \pm 0.6 \%$ and $27.3 \pm 3.0 \%$. The error bars are based on the EDS mappings at different selected areas. 

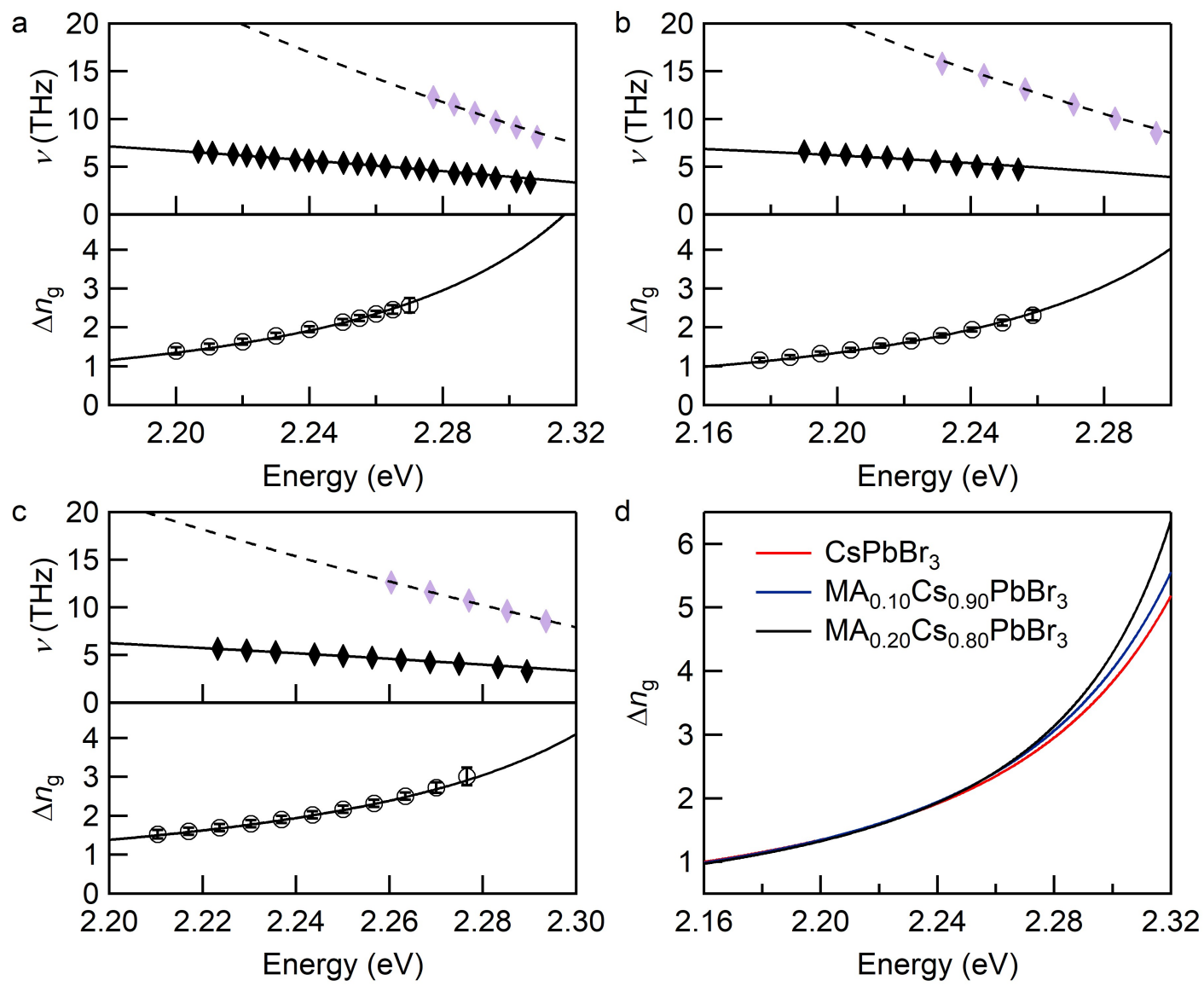

Figure S18. Global fitting results of the observed oscillation frequency and group index mismatch of $\mathrm{MA}_{\mathrm{x}} \mathrm{Cs}_{1-\mathrm{x}} \mathrm{PbBr}_{3}$ single crystals $(\mathrm{a}, \mathrm{x}=0 ; \mathrm{b}, \mathrm{x}=0.16 ; \mathrm{c}, \mathrm{x}=0.27)$. The diamond markers in upper panels are extracted from the two branches in the frequency-domain 2D-OKE spectra and the circles in lower panels are from the discontinuity in the time-domain spectra. (d) Group index mismatch spectra of different mixing ratio crystals.

Table S1. Lattice Parameters of MAPbBr 3 from SC-XRD.

\begin{tabular}{cccccc}
\hline$T(\mathrm{~K})$ & $a(\AA)$ & $c(\AA)$ & $b(\AA)$ & $e_{1}{ }^{\mathrm{s}}$ & $e_{6}^{\mathrm{s}}$ \\
\hline 100 & $7.9777(9)$ & $11.8530(15)$ & $8.5799(13)$ & $0.019589(5)$ & $0.057523(10)$
\end{tabular}




\begin{tabular}{llllll}
120 & $7.9941(11)$ & $11.8545(15)$ & $8.5752(13)$ & $0.018664(5)$ & $0.055477(11)$ \\
140 & $8.0105(12)$ & $11.8570(16)$ & $8.5693(14)$ & $0.017989(5)$ & $0.053320(11)$ \\
145 & $8.0172(18)$ & $11.8575(30)$ & $8.5671(20)$ & $0.017623(8)$ & $0.052460(16)$ \\
$150^{*}$ & $8.3204(15)$ & $11.7477(20)$ & & & \\
$152^{*}$ & $8.3231(18)$ & $11.7645(20)$ & & \\
$154^{*}$ & $8.3523(10)$ & $11.7369(15)$ & & \\
160 & $8.3034(11)$ & $11.8890(16)$ & & & \\
170 & $8.3079(12)$ & $11.8871(16)$ & & & \\
180 & $8.3124(12)$ & $11.8855(16)$ & & & \\
\hline
\end{tabular}

*The incommensurate phase lattice is averaged as a tetragonal phase lattice.

Table S2. Fitting Results of the Base Refractive Indices of $\mathrm{MAPbBr}_{3}$ and $\mathrm{MA}_{\mathbf{x}} \mathrm{Cs}_{1-\mathrm{x}} \mathrm{PbBr}_{3}$.

\begin{tabular}{lccccc}
\hline & $\begin{array}{c}p_{1} \\
\left(10^{-24} \mathrm{~Hz}^{-2}\right)\end{array}$ & $\begin{array}{c}p_{2} \\
\left(10^{-11} \mathrm{~Hz}^{-1}\right)\end{array}$ & $\begin{array}{c}p_{1} \\
\left(10^{6}\right)\end{array}$ & $\begin{array}{c}\left(10^{-11} \mathrm{~Hz}^{-1}\right) \\
\left(10^{6}\right)\end{array}$ \\
\hline $\mathrm{MAPbBr}_{3} 104.5 \mathrm{~K}$ & -35.03 & -4862 & 38.21 & -5075 & 28.04 \\
$\mathrm{MAPbBr}_{3} 127.4 \mathrm{~K}$ & -13.56 & -345.9 & 6.218 & -1103 & 5.866 \\
$\mathrm{MAPbBr}_{3} 149.0 \mathrm{~K}$ & -13.21 & -429.8 & 6.649 & -1143 & 6.122 \\
$\mathrm{MAPbBr}_{3} 149.4 \mathrm{~K}$ & -13.21 & -429.8 & 6.649 & -1143 & 6.122 \\
$\mathrm{MAPbBr}_{3} 151.8 \mathrm{~K}$ & -10.85 & -6942 & 42.46 & -4783 & 26.51 \\
$\mathrm{MAPbBr}_{3} 154.4 \mathrm{~K}$ & -10.85 & -6942 & 42.46 & -4783 & 26.51 \\
$\mathrm{MAPbBr}_{3} 159.7 \mathrm{~K}$ & 0.5783 & -1088 & 5.957 & -626.2 & 3.207 \\
$\mathrm{MAPbBr}_{3} 165.3 \mathrm{~K}$ & 0.5783 & -1088 & 5.957 & -626.2 & 3.207 \\
$\mathrm{MAPbBr}_{3} 170.8 \mathrm{~K}$ & 0.5783 & -1088 & 5.957 & -626.2 & 3.207
\end{tabular}




\begin{tabular}{cccccc}
$\mathrm{CsPbBr}_{3}$ & -6.039 & -1089 & 8.621 & -1049 & 5.851 \\
$\mathrm{MA}_{0.16} \mathrm{Cs}_{0.84} \mathrm{PbBr}_{3}$ & -6.039 & -1089 & 8.621 & -1049 & 5.851 \\
$\mathrm{MA}_{0.27} \mathrm{Cs}_{0.73} \mathrm{PbBr}_{3}$ & -6.039 & -1089 & 8.621 & -1049 & 5.851 \\
\hline
\end{tabular}

Table S3. Fitting Results of Parameters in Refractive Indices of MAPbBr3 at Different Temperatures and $\mathrm{MA}_{\mathrm{x}} \mathrm{Cs}_{1-\mathrm{x}} \mathrm{PbBr}_{3}$.

\begin{tabular}{|c|c|c|c|c|c|c|c|c|c|}
\hline & $a_{1}$ & $\begin{array}{c}a_{2} \\
\left(10^{12}\right. \\
\mathrm{Hz})\end{array}$ & $\begin{array}{c}a_{3} \\
\left(10^{56}\right. \\
\left.\mathrm{Hz}^{4}\right)\end{array}$ & $\begin{array}{c}a_{4} \\
\left(10^{12} \mathrm{~Hz}\right)\end{array}$ & $a_{5}$ & $a_{6}$ & $\begin{array}{c}a_{7} \\
\left(10^{12} \mathrm{~Hz}\right)\end{array}$ & $a_{8}$ & $a_{9}$ \\
\hline \multicolumn{10}{|l|}{$\mathrm{MAPbBr}_{3}$} \\
\hline $104.5 \mathrm{~K}$ & 0.9057 & -0.5132 & 12.52 & 561.7 & 0.1703 & 1.290 & -0.9879 & -0.4888 & 726.7 \\
\hline \multicolumn{10}{|l|}{$\mathrm{MAPbBr}_{3}$} \\
\hline $127.4 \mathrm{~K}$ & 0.7093 & -1.082 & 11.56 & 561.9 & 0.4826 & 1.173 & 0.2442 & -0.2904 & 629.5 \\
\hline \multicolumn{10}{|l|}{$\mathrm{MAPbBr}_{3}$} \\
\hline $149.0 \mathrm{~K}$ & 1.020 & 0.3770 & 20.00 & 563.5 & -0.006940 & 1.129 & 0.04821 & -0.2205 & 696.4 \\
\hline \multicolumn{10}{|l|}{$\mathrm{MAPbBr}_{3}$} \\
\hline $149.4 \mathrm{~K}$ & 0.8826 & 0.2950 & 18.34 & 563.5 & 0.2096 & 1.115 & -0.08612 & -0.1931 & 716.4 \\
\hline \multicolumn{10}{|l|}{$\mathrm{MAPbBr}_{3}$} \\
\hline $151.8 \mathrm{~K}$ & 0.7717 & -6.347 & -0.7856 & 563.0 & 0.3919 & 1.181 & -1.833 & -0.3182 & 479.1 \\
\hline \multicolumn{10}{|l|}{$\mathrm{MAPbBr}_{3}$} \\
\hline $154.4 \mathrm{~K}$ & 0.7856 & -7.542 & -2.967 & 561.0 & 0.3663 & 1.190 & -1.588 & -0.3392 & 678.6 \\
\hline \multicolumn{10}{|l|}{$\mathrm{MAPbBr}_{3}$} \\
\hline $159.7 \mathrm{~K}$ & 0.2055 & -10.34 & -14.90 & 548.5 & 1.556 & 1.247 & -2.000 & -0.4928 & 247.7 \\
\hline \multicolumn{10}{|l|}{$\mathrm{MAPbBr}_{3}$} \\
\hline $165.3 \mathrm{~K}$ & 0.2239 & -14.90 & -14.36 & 544.3 & 1.559 & 1.220 & -2.000 & -0.4507 & 423.6 \\
\hline
\end{tabular}




\begin{tabular}{cccccccccc}
$\mathrm{MAPbBr}_{3}$ & & & & & & & & & \\
$170.8 \mathrm{~K}$ & 0.3104 & -2.000 & -0.6055 & 570.0 & 1.351 & 1.062 & -0.4469 & -0.1275 & 329.7 \\
$\mathrm{CsPbBr}_{3}$ & 0.7439 & 0.1895 & -13.21 & 586.8 & 0.4502 & 1.109 & -0.2000 & -0.1911 & 661.2 \\
$\mathrm{MA}_{0.16} \mathrm{Cs}_{0.84}$ & & & & & & & & & \\
$\mathrm{PbBr}_{3}$ & 0.7255 & 0.3635 & -9.258 & 577.5 & 0.4856 & 1.125 & -0.1996 & -0.2295 & 861.0 \\
$\mathrm{MA}_{0.27} \mathrm{Cs}_{0.73}$ & & & & & & & & & \\
$\mathrm{PbBr}_{3}$ & 0.6723 & -0.1231 & -13.99 & 577.5 & 0.5787 & 1.1120 & -0.2000 & -0.2166 & 785.5 \\
\hline
\end{tabular}

Table S4. Lattice Parameters of Mixed-Cation MAxCs1-xPbBr3 from SC-XRD.

\begin{tabular}{ccccc}
\hline $\mathrm{x}$ & $a(\AA)$ & $b(\AA)$ & $c(\AA)$ & Volume $\left(\AA^{3}\right)$ \\
\hline 0 & $8.2062(7)$ & $8.2438(7)$ & $11.7524(8)$ & $795.05(11)$ \\
0 & $8.2070(7)$ & $8.2550(8)$ & $11.7590(8)$ & $796.66(12)$ \\
0.16 & $8.2234(10)$ & $8.2486(14)$ & $11.7192(17)$ & $794.90(20)$ \\
0.16 & $8.2262(18)$ & $8.2465(12)$ & $11.7020(20)$ & $793.80(20)$ \\
0.27 & $8.2044(13)$ & $8.2489(11)$ & $11.7357(19)$ & $794.20(20)$ \\
0.27 & $8.2052(7)$ & $8.2517(7)$ & $11.7521(8)$ & $795.70(11)$ \\
0.27 & $8.2191(10)$ & $8.2516(10)$ & $11.7176(17)$ & $794.69(18)$ \\
\hline
\end{tabular}

Supplementary Note 1. Global fitting of energy-dependent refractive index and birefringence

We obtain the fast and slow refractive indices $\left(n_{\mathrm{f}}\right.$ and $\left.n_{\mathrm{s}}\right)$ and the birefringence $(\Delta n)$ by globally fitting the observed pump-probe co-propagation time $t$ and the oscillation frequency $\omega_{\text {obs. The }}$ method is recently demonstrated by Huber et al. ${ }^{7}$ A base refractive index $n_{0}$ is first fitted to the published data for $\mathrm{MAPbBr}_{3}$ single crystals at different temperatures ${ }^{4}$ and $\mathrm{CsPbBr}_{3}$ single $\mathrm{crystal}^{4}$ or thin film ${ }^{8}$ at room temperature 


$$
n_{0}(v)=\frac{p_{1} v^{2}+p_{2} v+p_{3}}{v^{2}+q_{1} v+q_{2}}
$$

The fitting results of $p_{1}, p_{2}, p_{3}, q_{1}, q_{2}$ are listed in Table S2 with $v=\omega / 2 \pi$. Then the refractive index model function is parameterized by scaling and shifting the $n_{0}$ and used to calculate $n_{\mathrm{f}}$ and $n_{\mathrm{s}}$ :

$$
\begin{gathered}
n_{\mathrm{f}}(v)=a_{1}\left(n_{0}\left(v+a_{2}\right)+\frac{a_{3}}{\left(v+a_{2}\right)^{4}-a_{4}^{4}}\right)+a_{5} \\
n_{\mathrm{s}}(v)=a_{6} n_{\mathrm{f}}\left(v+a_{7}\right)+a_{8}+a_{9} \frac{q_{3}^{2}}{v^{2}-q_{3}^{2}}
\end{gathered}
$$

where $a_{\mathrm{i}}$ are parameters to fit, the quartic term accounts for the steep resonance response and a phonon oscillator term at frequency $q_{3}=2 \mathrm{THz}$ is added. The group index $n_{\mathrm{g}}$ is derived from the refractive index $n$ via $n_{\mathrm{g}}(\omega)=n(\omega)+\omega \partial n(\omega) / \partial \omega$ and the $\Delta n_{\mathrm{g}}$ is the difference between pump and probe pulse group indices. Therefore, based on the refractive index model functions, the derived

group index and Eq. 1 and Eq. 2 in the main text, we use the genetic algorithm to globally fit the experimental values of $\Delta n_{\mathrm{g}}$ and $\omega_{\mathrm{obs}}$ from the 2D-OKE. Both low- and high-frequency branches are fitted for $\mathrm{MAPbBr}_{3}$ at $T=104.5 \sim 154.4 \mathrm{~K}$ and for $\mathrm{MA}_{1-\mathrm{x}} \mathrm{Cs}_{\mathrm{x}} \mathrm{PbBr}_{3}$ and only the low-frequency branch is fitted for $\mathrm{MAPbBr}_{3}$ at $T=159.7 \sim 170.8 \mathrm{~K}$. The fitting results are listed in Table S3. Note that the observed FT-frequency is shifted by a factor of 0.31 for the both branches in $\mathrm{MAPbBr}_{3}$ and only for the high-frequency branch in $\mathrm{MA}_{1-\mathrm{x}} \mathrm{Cs}_{\mathrm{x}} \mathrm{PbBr}_{3}$ data. The factor of 0.31 is obtained based on the 2D-OKE simulation. ${ }^{7}$

\section{Supplementary Note 2. Correlation between the birefringence and lattice constants}

Here we establish the correlation between the birefringence and lattice parameter contrast for the orthorhombic (Pnma) and tetragonal $(I 4 / \mathrm{mcm})$ phase of $\mathrm{MAPbBr}_{3}$. The relationship can be derived from the relative dielectric impermeability tensor $\delta B_{m}$ as a function of spontaneous strain $e_{\mathrm{n}}{ }^{s}$ and photoelastic moduli $p_{\mathrm{mn}}$ :

$$
\delta B_{m}=p_{m n} e_{n}^{S}
$$


The orthorhombic and tetragonal can be transformed from the pseudo-cubic phase by applying the uniaxial and biaxial tensile stress, thus the transformation of mmmFm $3 \mathrm{~m}$ and $4 / \mathrm{mmmFm} 3 \mathrm{~m}$ is used to express the spontaneous strain tensor for the two phases, respectively.

For the orthorhombic ( $P$ nma) phase, the $\delta B_{m}$ tensor elements are written using the reduced-suffix notation of $\mathrm{Nye}^{9}$

$$
\begin{gathered}
\delta B_{1}=p_{11}\left(e_{1}^{S}+\Delta\right)+p_{12}\left(e_{1}^{S}+\Delta\right)+p_{12}\left(-2 e_{1}^{S}+\Delta\right) \\
\delta B_{2}=p_{12}\left(e_{1}^{s}+\Delta\right)+p_{11}\left(e_{1}^{s}+\Delta\right)+p_{12}\left(-2 e_{1}^{s}+\Delta\right) \\
\delta B_{3}=p_{12}\left(e_{1}^{S}+\Delta\right)+p_{12}\left(e_{1}^{s}+\Delta\right)+p_{11}\left(-2 e_{1}^{S}+\Delta\right) \\
\delta B_{3}=\delta B_{5}=0 \\
\delta B_{6}=p_{44} e_{6}^{S}
\end{gathered}
$$

where the strains are divided into volume dilation $\Delta$, shear $\left(e_{6}\right)$ and elongations $\left(e_{1}=e_{2} \neq e_{3}\right)$ and the spontaneous strain tensor elements $e_{\mathrm{n}}^{s}$ are related to the normal strain components $e_{\mathrm{n}}$ by $e_{1}^{s}=$ $\frac{1}{3}\left(e_{1}-e_{3}\right)$ and $e_{6}^{s}=e_{6} \cdot{ }^{10}$ In terms of the orthorhombic axes, the strains are given by

$$
e_{1}=\frac{2 \xi-a_{0}-b_{0}}{2 \xi}, e_{3}=\frac{\sqrt{2} \xi-c_{0}}{\sqrt{2} \xi}, e_{6}=\frac{b_{0}-a_{0}}{2 \xi}
$$

where $\xi=\left(a_{0} b_{0} c_{0} / \sqrt{2}\right)^{1 / 3}$, the cube root of the volume of the orthorhombic cell. The dielectric impermeability is related to the refractive indices by $B=\frac{1}{n^{2}}$ and hence $\delta n_{i}=-\left(\frac{n_{i}^{3}}{2}\right) \delta B_{i}$. To a reasonable approximation we can replace $n_{\mathrm{i}}$ by a constant $n_{0}$ corresponding to the refractive index of the pseudo-cubic case without any strain. ${ }^{11,12}$ Then the three possible orthorhombic birefringences are

$$
\begin{gathered}
\Delta n_{a b}=\delta n_{1}-\delta n_{2}=-n_{0}^{3} p_{44} e_{6}^{s} \\
\Delta n_{b c}=\delta n_{2}-\delta n_{3}=-\frac{1}{2} n_{0}^{3}\left[3\left(p_{11}-p_{12}\right) e_{1}^{s}-p_{44} e_{6}^{s}\right] \\
\Delta n_{a c}=\delta n_{1}-\delta n_{3}=-\frac{1}{2} n_{0}^{3}\left[3\left(p_{11}-p_{12}\right) e_{1}^{s}+p_{44} e_{6}^{s}\right] .
\end{gathered}
$$

In the orthorhombic phase of $\mathrm{MAPbBr}_{3}$, the linear relationship is found $e_{6}^{s}=k \cdot e_{1}^{s}+\beta$ (Fig. S5). 
Therefore, the birefringence of interest is

$$
\Delta n_{a c}=-\frac{1}{2} n_{0}^{3}\left\{\left[3\left(p_{11}-p_{12}\right)+k p_{44}\right] e_{1}^{S}+p_{44} \beta\right\} .
$$

For orthorhombic $\mathrm{MAPbBr}_{3}$, the value of $e_{1}^{s}$ is approximately given by $e_{1}^{s}=c / a-1,{ }^{12}$ therefore, the linear relationship can be derived respectively:

$$
\frac{2\left|\Delta n_{a c}\right|}{n_{0}^{3}(c / a-1)}=\frac{p_{44} \beta}{(c / a-1)}+\left[3\left(p_{11}-p_{12}\right)+k p_{44}\right]
$$

For the tetragonal phase $(I 4 / \mathrm{mcm})$, the $\delta B_{m}$ tensor elements are

$$
\begin{gathered}
\delta B_{1}=p_{11} e_{1}^{S}+p_{12} e_{1}^{S}+p_{12} e_{3}^{S} \\
\delta B_{2}=p_{12} e_{1}^{S}+p_{11} e_{1}^{S}+p_{12} e_{3}^{S} \\
\delta B_{3}=p_{12} e_{1}^{S}+p_{12} e_{1}^{S}+p_{11} e_{3}^{S} \\
\delta B_{4}=\delta B_{5}=\delta B_{6}=0 .
\end{gathered}
$$

Then the interested birefringence $\Delta n_{a c}=\delta n_{1}-\delta n_{3}=-\frac{1}{2} n_{0}^{3}\left[3\left(p_{11}-p_{12}\right)\left(e_{1}^{s}-e_{3}^{s}\right)\right]$ can be rearranged as

$$
\frac{2\left|\Delta n_{a c}\right|}{n_{0}^{3}(c / a-1)}=\frac{3 \beta^{\prime}\left(p_{11}-p_{12}\right)}{(c / a-1)}+3 k^{\prime}\left(p_{11}-p_{12}\right)
$$

by making the approximation of $\left(e_{1}^{S}-e_{3}^{S}\right)=k^{\prime}(c / a-1)+\beta^{\prime} .^{12}$

\section{Supplementary Note 3. Landau theory and energy gap anomaly near phase transitions}

Here we discuss the thermodynamic property, i.e., the free energy, based on the Landau theory for phase transition by using the polarization as the order parameter. ${ }^{13}$ The presence of spontaneous polarization $(P)$ or ferroelectricity in three-dimensional LHPs is still under debate due to the complexity in the dynamic symmetry breaking and the chemical composition heterogeneity or ion diffusions. ${ }^{14-22}$ Note that, the order parameter here is treated in general or as the subsystem associated with the polar vibrational mode. The Landau free energy function can be written as 


$$
F=F_{0}+\frac{1}{2} \alpha P^{2}+\frac{1}{4} \beta P^{4}+\frac{1}{6} \gamma P^{6}
$$

where coefficients $\alpha>0, \beta<0, \gamma>0$ for the first-order and $\alpha<0, \beta>0, \gamma>0$ for second-order phase transition, and $F_{0}$ is the free energy for $P=0$. The temperature-dependence of polarization order parameter (Fig. 3d) can be derived by $\frac{\partial F}{\partial P}=0$

for first-order: $P^{2}=\frac{-\beta+\sqrt{\beta^{2}-4 \alpha\left(T-T_{\mathrm{c}}\right) \gamma}}{2 \gamma}$ when $T<T_{\mathrm{c}} ; P^{2}=0, \frac{-\beta+\sqrt{\beta^{2}-4 \alpha\left(T-T_{\mathrm{c}}\right) \gamma}}{2 \gamma}$ when $T_{\mathrm{c}}<T<T_{1}$; $P^{2}=0$ when $T_{1}<T$; where $T_{1}=\frac{\beta^{2}}{4 \alpha \gamma}+T_{\mathrm{c}}$;

for second-order: $P^{2}=\frac{\alpha\left(T-T_{\mathrm{c}}\right)}{\beta}$ when $T<T_{\mathrm{c}}, P=0$ when $T>T_{\mathrm{c}}$.

Then the energy gap anomaly can be derived by considering the free energy of the electron subsystem $\left(F_{\mathrm{e}}\right)$, which is usually small compared with the free energy of the lattice and that the phase transition is associated with instability in the lattice vibrations. ${ }^{23}$ The expression of $F_{\mathrm{e}}=$ $\sum_{i} N_{i} E_{i}\left(T, P, \sigma_{k}\right)$ is a summation over energy levels of the electron subsystem $E_{\mathrm{i}}$ and the respective electron (hole) concentrations $N_{\mathrm{i}}$ in the crystal, where $\sigma_{\mathrm{k}}$ is the component of the mechanical stress tensor. It is noted that $F_{\mathrm{e}}$ describes the contribution from free electrons and holds in the shallow local levels. For a n-type semiconductor, we can represent $F_{\mathrm{e}}$ as

$$
F_{\mathrm{e}}=N \tilde{E} \cong N\left(E_{\mathrm{g}}-\mu_{1}-\mu_{2}\right)
$$

where $\mu_{1}, \mu_{2}$ is the chemical potential of electron and hole; $N$ is the concentration of free electrons; $E_{\mathrm{g}}$ is the intrinsic bandgap. When the crystal is not subject to external mechanical stresses, we can simplify the expression of the energy gap

$$
\tilde{E}=E_{0}+\frac{1}{2} a P^{2}+\frac{1}{4} b P^{4}+\frac{1}{6} c P^{6}
$$

where $E_{0}$ is the energy gap with $P=0 ; \mathrm{a}, \mathrm{b}$ and c are the coefficients related but not equal to $\alpha, \beta$, and $\gamma$. Thus the discontinuity in the first-order phase transition is approximately

$$
\Delta \tilde{E} \cong \frac{a}{4 \pi} C \cdot \Delta S
$$


in which $C$ is the Curie-Weiss constant and $\Delta S$ is entropy change. However, it disappears in a second-order transition $\Delta \tilde{E}=0$ and there will be a discontinuity in the derivatives of the energy gap. It is worth to point out that the energy gap anomaly can be derived in general using the model of Slater and there is such a discontinuity in the energy gap or its derivatives in nonferroelectric transitions..$^{24,25}$

\section{Supplementary Note 4. Collective modes in incommensurate (INC) phase}

The thermodynamic potential of INC phase just below transition temperature $T_{\mathrm{i}}$ where a single plane wave approximation is valid can be written as

$$
\phi_{I N C}=\alpha_{k} Q_{k} Q_{k}^{*}+\frac{\beta}{2}\left(Q_{k} Q_{k}^{*}\right)^{2}
$$

where $Q_{\mathrm{k}}$ denotes the complex normal mode from the soft branch. ${ }^{26}$ Note that the high order terms including the interaction with polarization modes are omitted here for simplicity. The increase of potential in terms of anharmonic interactions of soft modes around the equilibrium INC-structure becomes

$$
\delta \phi=\sum\left[\left(\alpha_{k_{i} \pm q}+2 \beta \eta_{i}^{2}\right) Q_{k_{i} \pm q} Q_{k_{i} \pm q}^{*}+\beta \eta_{i}^{2}\left(Q_{-k_{i} \pm q} Q_{-k_{i} \pm q}^{*}+\text { c.c. }\right)\right]
$$

where the anharmonic term $\eta_{i}$ denotes the amplitude of the frozen-in mode with wave vector $k_{i}$. Now the soft-branch modes with $\left(k_{i}+q\right)$ and $\left(-k_{i}+q\right)$ are coupled and are degenerate. The $Q$-part of $\delta \phi$ can be diagonalized as

$$
\delta \phi=\sum_{q}\left(\omega_{q}^{2} \phi_{q} \phi_{q}^{*}+\Omega_{q}^{2} A_{q} A_{q}^{*}\right)
$$

by introducing symmetric and antisymmetric combination of coupled modes

$$
\begin{aligned}
& A_{q}=\frac{1}{\sqrt{2}}\left(Q_{k_{i}+q}+Q_{-k_{i}+q}\right) \\
& \varphi_{q}=\frac{1}{\sqrt{2}}\left(Q_{k_{i}+q}-Q_{-k_{i}+q}\right) .
\end{aligned}
$$

The in-phase mode $A_{q}$ (amplitudon) with eigenvalue of $\Omega_{q}^{2}=2 \beta \eta_{i}^{2}+\kappa q^{2}$ and the out-of-phase mode $\varphi_{q}$ (phason) with eigenvalue of $\omega_{q}^{2}=\kappa q^{2}$ describes the atom displacements related to the amplitude modulation of the static distortion and the phase modulation of the frozen INC wave, respectively. ${ }^{27}$ It should be pointed out that they are normal modes of the INC phase only for small 
$q$ where the soft branch can be approximated by a parabola. Far below $T_{\mathrm{i}}$ where the INC-structure becomes domain-like, the continuous model is more convenient than the mode expression. ${ }^{28-30}$ The amplitudon with $q=0$ is a totally symmetric Raman active mode, which has been observed previously. ${ }^{31,32}$ However, because the phason frequency approaches zero towards the zone center and the damping is largely independent on momentum, ${ }^{33}$ the phason is usually very close to the strong Rayleigh scattering and too overdamped for clear identification. ${ }^{34}$

In the ferroelectric $\mathrm{K}_{2} \mathrm{SeO}_{4}$, the phase transition behaviors are studied by Iizumi et al, ${ }^{27}$ in which a continuous transition at $T_{\mathrm{i}} \sim 128 \mathrm{~K}$ from the paraelectric parent phase (Pnam, $\mathrm{D}_{2 \mathrm{~h}}{ }^{16}$ ) into an INC phase is followed by a lock-in (likely first order) transition at $T_{\mathrm{C}} \sim 93 \mathrm{~K}$ into a commensurate phase $\left(P\right.$ na2 $\left.{ }_{1}, \mathrm{C}_{2 v}{ }^{9}\right)$. The neutron inelastic scattering reveals that the transition into INC phase is due to softening of a normal mode with the wave vector near the commensurate point of the Brillouin zone $k_{c}=\frac{1}{3} a^{*}$, where $a^{*}$ is the basic vector of the reciprocal lattice in the a direction.

\section{Supplementary Note 5. Twin structure and incommensurate wave vector analysis}

As shown in our data and the previous report ${ }^{34}$, the two-fold twin structure develops when the crystal undergoes transition from the tetragonal phase and additional symmetric splitting appears in the reflection pattern. The strong twinning reflection will interfere the full structural characterization of the incommensurate modulation, therefore, we refine the twinned basic structure by omitting satellite reflections from the data set. Since the data are collected successively across the phase transition, it is plausible to use a common orientation matrix to relate the phases. The tetragonal direct basis is transformed to the incommensurate phase by the matrix:

$$
\left(\begin{array}{l}
a \\
b \\
c
\end{array}\right)_{i n c}=\left(\begin{array}{rrc}
-\frac{1}{2} & \frac{1}{2} & -\frac{1}{2} \\
-\frac{1}{2} & \frac{1}{2} & \frac{1}{2} \\
1 & 1 & 0
\end{array}\right)\left(\begin{array}{l}
a \\
b \\
c
\end{array}\right)_{t I}
$$

A second twin-related domain is given by the matrix: 


$$
\left(\begin{array}{l}
a \\
b \\
c
\end{array}\right)_{i n c}=\left(\begin{array}{ccc}
-\frac{1}{2} & -\frac{1}{2} & \frac{1}{2} \\
\frac{1}{2} & \frac{1}{2} & \frac{1}{2} \\
-1 & 1 & 0
\end{array}\right)\left(\begin{array}{l}
a \\
b \\
c
\end{array}\right)_{t I}
$$

Both transformation matrices are rounded to omit the changes in cell volume. The orientation matrices after twin lattice determination are applied to the data set and the incommensurate modulation wave vector is found with tolerance $\leq 0.15$ for the lattice refinement. For the pure $\mathrm{MAPbBr}_{3}$, this method is used for the data of 148 to $155 \mathrm{~K}$ and a significant drop in the indexed percentage shows for the data with temperature $\leq 147 \mathrm{~K}$, indicating the transition into the orthorhombic phase. The same method is applied to the mixed-cation $\mathrm{Cs}_{0.07} \mathrm{MA}_{0.93} \mathrm{PbBr}_{3}$ perovskite to extract the $q_{\text {inc }}$ vector and determine the incommensurate phase temperature window.

\section{Reference}

(1) Liu, F.; Wang, F.; Hansen, K. R.; Zhu, X.-Y. Bimodal Bandgaps in Mixed Cesium Methylammonium Lead Bromide Perovskite Single Crystals. J. Phys. Chem. C 2019, 123 (23), 14865-14870.

(2) Maehrlein, S. F.; Joshi, P. P.; Huber, L.; Wang, F.; Cherasse, M.; Liu, Y.; Juraschek, D. M.; Mosconi, E.; Meggiolaro, D.; Angelis, F. De; et al. Decoding Ultrafast Polarization Responses in Lead Halide Perovskites by the Two-Dimensional Optical Kerr Effect. Proc Natl Acad Sci USA 2021, 118 (7), e2022268118.

(3) Hamm, P.; Zanni, M. Concepts and Methods of 2D Infrared Spectroscopy; Cambridge University Press: Cambridge, 2011.

(4) Guo, Y.; Yaffe, O.; Hull, T. D.; Owen, J. S.; Reichman, D. R.; Brus, L. E. Dynamic Emission Stokes Shift and Liquid-like Dielectric Solvation of Band Edge Carriers in Lead-Halide Perovskites. Nat. Commun. 2019, 10 (1), 1175.

(5) Leguy, A. M. A.; Goñi, A. R.; Frost, J. M.; Skelton, J.; Brivio, F.; Rodríguez-Martínez, X.; Weber, O. J.; Pallipurath, A.; Alonso, M. I.; Campoy-Quiles, M.; et al. Dynamic Disorder, Phonon Lifetimes, and the Assignment of Modes to the Vibrational Spectra of Methylammonium Lead Halide Perovskites. Phys. Chem. Chem. Phys. 2016, 18 (39), 27051-27066.

(6) Zhang, L.; Yang, X.; Jiang, Q.; Wang, P.; Yin, Z.; Zhang, X.; Tan, H.; Yang, Y. (Michael); 
Wei, M.; Sutherland, B. R.; et al. Ultra-Bright and Highly Efficient Inorganic Based Perovskite Light-Emitting Diodes. Nat. Commun. 2017, 8 (1), 15640.

(7) Huber, L.; Maehrlein, S. F.; Wang, F.; Liu, Y.; Zhu, X.-Y. The Ultrafast Kerr Effect in Anisotropic and Dispersive Media. J. Chem. Phys. 2021, 154, 094202.

(8) Schlaus, A. P.; Spencer, M. S.; Miyata, K.; Liu, F.; Wang, X.; Datta, I.; Lipson, M.; Pan, A.; Zhu, X.-Y. How Lasing Happens in $\mathrm{CsPbBr}_{3}$ Perovskite Nanowires. Nat. Commun. 2019, 10 (1), 265.

(9) Nye, J. F. Physical Properties of Crystals; Oxford University Press: New York, 1957.

(10) Aizu, K. Determination of the State Parameters and Formulation of Spontaneous Strain for Ferroelastics. J. Phys. Soc. Japan 1970, 28 (3), 706-716.

(11) Stadnicka, K.; Glazer, A. M. Birefringence and Lattice Parameters of Dicalcium Barium Acrylate and Related Materials. Phase Transitions 1982, 2 (4), 293-308.

(12) Mabud, S. A.; Glazer, A. M. Lattice Parameters and Birefringence in $\mathrm{PbTiO}_{3}$ Single Crystals. J. Appl. Crystallogr. 1979, 12 (1), 49-53.

(13) Rabe, K. M.; Ahn, C. H.; Triscone, J.-M. Physics of Ferroelectrics; Topics in Applied Physics; Springer Berlin Heidelberg: Berlin, Heidelberg, 2007; Vol. 105.

(14) Schulz, A. D.; Röhm, H.; Leonhard, T.; Wagner, S.; Hoffmann, M. J.; Colsmann, A. On the Ferroelectricity of $\mathrm{CH}_{3} \mathrm{NH}_{3} \mathrm{PbI}_{3}$ Perovskites. Nat. Mater. 2019, 18 (10), 1050.

(15) Liu, Y.; Collins, L.; Proksch, R.; Kim, S.; Watson, B. R.; Doughty, B.; Calhoun, T. R.; Ahmadi, M.; Ievlev, A. V; Jesse, S.; et al. Reply to: On the Ferroelectricity of $\mathrm{CH}_{3} \mathrm{NH}_{3} \mathrm{PbI}_{3}$ Perovskites. Nat. Mater. 2019, 18 (10), 1051-1053.

(16) Röhm, H.; Leonhard, T.; Hoffmann, M. J.; Colsmann, A. Ferroelectric Domains in Methylammonium Lead Iodide Perovskite Thin-Films. Energy Environ. Sci. 2017, 10 (4), 950955.

(17) Beilsten-Edmands, J.; Eperon, G. E.; Johnson, R. D.; Snaith, H. J.; Radaelli, P. G. NonFerroelectric Nature of the Conductance Hysteresis in $\mathrm{CH}_{3} \mathrm{NH}_{3} \mathrm{PbI}_{3}$ Perovskite-Based Photovoltaic Devices. Appl. Phys. Lett. 2015, 106 (17), 173502.

(18) G, S.; Mahale, P.; Kore, B. P.; Mukherjee, S.; Pavan, M. S.; De, C.; Ghara, S.; Sundaresan, A.; Pandey, A.; Guru Row, T. N.; et al. Is $\mathrm{CH}_{3} \mathrm{NH}_{3} \mathrm{PbI}_{3}$ Polar? J. Phys. Chem. Lett. 2016, 7 (13), 2412-2419.

(19) Gómez, A.; Wang, Q.; Goñi, A. R.; Campoy-Quiles, M.; Abate, A. Ferroelectricity-Free 
Lead Halide Perovskites. Energy Environ. Sci. 2019, 12 (8), 2537-2547.

(20) Liu, Y.; Collins, L.; Proksch, R.; Kim, S.; Watson, B. R.; Doughty, B.; Calhoun, T. R.; Ahmadi, M.; Ievlev, A. V; Jesse, S.; et al. Chemical Nature of Ferroelastic Twin Domains in $\mathrm{CH}_{3} \mathrm{NH}_{3} \mathrm{PbI}_{3}$ Perovskite. Nat. Mater. 2018, 17 (11), 1013-1019.

(21) Röhm, H.; Leonhard, T.; Hoffmann, M. J.; Colsmann, A. Ferroelectric Poling of Methylammonium Lead Iodide Thin Films. Adv. Funct. Mater. 2020, 30 (5), 1908657.

(22) Rakita, Y.; Bar-Elli, O.; Meirzadeh, E.; Kaslasi, H.; Peleg, Y.; Hodes, G.; Lubomirsky, I.; Oron, D.; Ehre, D.; Cahen, D. Tetragonal $\mathrm{CH}_{3} \mathrm{NH}_{3} \mathrm{PbI}_{3}$ Is Ferroelectric. Proc. Natl. Acad. Sci. 2017, 114 (28), E5504--E5512.

(23) Fridkin, V. M. Photoferroelectrics; Springer Series in Solid-State Sciences; Springer Berlin Heidelberg: Berlin, Heidelberg, 1979; Vol. 9.

(24) Slater, J. C. Introduction to Chemical Physics; McGraw-Hill book company, inc., 1939.

(25) Keyes, R. W. The Effects of Elastic Deformation on the Electrical Conductivity of Semiconductors; SEITZ, F., TURNBULL, D. B. T.-S. S. P., Eds.; Academic Press, 1960; Vol. 11, pp 149-221.

(26) Dvorak, V.; Petzelt, J. Infrared and Raman Activity of Soft Modes in the Incommensurate Structure. J. Phys. C Solid State Phys. 1978, 11 (23), 4827-4835.

(27) Iizumi, M.; Axe, J. D.; Shirane, G.; Shimaoka, K. Structural Phase Transformation in $\mathrm{K}_{2} \mathrm{SeO}_{4}$. Phys. Rev. B 1977, 15 (9), 4392-4411.

(28) Ishibashi, Y. Incommensurate Phase Transitions in Ferroelectrics. Ferroelectrics 1980, 24 (1), 119-126.

(29) Bruce, A. D.; Cowley, R. A.; Murray, A. F. The Theory of Structurally Incommensurate Systems. II. Commensurate-Incommensurate Phase Transitions. J. Phys. C Solid State Phys. 1978, 11 (17), 3591-3608.

(30) Bruce, A. D.; Cowley, R. A. The Theory of Structurally Incommensurate Systems. III. The Fluctuation Spectrum of Incommensurate Phases. J. Phys. C Solid State Phys. 1978, 11 (17), 36093630.

(31) Wada, M.; Sawada, A.; Ishibashi, Y.; Takagi, Y. Raman Scattering Spectra of $\mathrm{K}_{2} \mathrm{SeO}_{4}$. J. Phys. Soc. Japan 1977, 42 (4), 1229-1234.

(32) Delahaigue, A.; Khelifa, B.; Jouve, P. Soft Mode in Thiourea by Raman Measurements. Phys. Status Solidi 1975, 72 (2), 585-589. 
(33) Cummins, H. Z. Experimental Studies of Structurally Incommensurate Crystal Phases. Phys. Rep. 1990, 185 (5), 211-409.

(34) Guo, Y.; Yaffe, O.; Paley, D. W.; Beecher, A. N.; Hull, T. D.; Szpak, G.; Owen, J. S.; Brus, L. E.; Pimenta, M. A. Interplay between Organic Cations and Inorganic Framework and Incommensurability in Hybrid Lead-Halide Perovskite $\mathrm{CH}_{3} \mathrm{NH}_{3} \mathrm{PbBr}_{3}$. Phys. Rev. Mater. 2017, 1 (4), 42401. 